\title{
THE GEOMETRY OF GRASSMANNIAN MANIFOLDS AND BERNSTEIN TYPE THEOREMS FOR HIGHER CODIMENSION
}

\author{
J. JOST, Y. L. XIN AND LING YANG
}

\begin{abstract}
We identify a region $\mathbb{W}_{\frac{1}{3}}$ in a Grassmann manifold $\mathbf{G}_{n, m}$, not covered by a usual matrix coordinate chart, with the following important property. For a complete $n$-submanifold in $\mathbb{R}^{n+m}(n \geq 3, m \geq 2)$ with parallel mean curvature whose image under the Gauss map is contained in a compact subset $K \subset \mathbb{W}_{\frac{1}{3}} \subset \mathbf{G}_{n, m}$, we can construct strongly subharmonic functions and derive a priori estimates for the harmonic Gauss map. While we do not know yet how close our region is to being optimal in this respect, it is substantially larger than what could be achieved previously with other methods. Consequently, this enables us to obtain substantially stronger Bernstein type theorems in higher codimension than previously known.
\end{abstract}

\section{INTRODUCTION}

The classical Bernstein theorem states that any complete minimal graph $M^{2}$ in $\mathbb{R}^{3}$ has to be an affine plane. Equivalently, all tangent planes are parallel to each other, and the Gauss map $\gamma: M \rightarrow S^{2}$ is constant. This scheme extends to higher dimensions, that is, minimal graphs in $\mathbb{R}^{n+1}$, although the result becomes somewhat weaker for $n \geq 7$. In this work, we are interested in such a scheme for higher codimensional minimal submanifolds of Euclidean space.

We now make this more precise. Let $M^{n}$ be a complete $n$-dimensional submanifold in Euclidean space $\mathbb{R}^{n+m}$. The Grassmann manifold $\mathbf{G}_{n, m}$ is the target manifold of the Gauss map $\gamma: M \rightarrow \mathbf{G}_{n, m}$ that assigns to each point of $M$ the direction of its tangent space in $\mathbb{R}^{n+m}$. By the Ruh-Vilms theorem [20] the Gauss map is a harmonic map into the Grassmann manifold iff $M$ has parallel mean curvature. In particular, the Gauss map is harmonic for minimal submanifolds of Euclidean space. When $m=1$, the Grassmann manifold reduces to the sphere $S^{n}$, and investigating the Gauss map has identified many conditions under which a minimal submanifold has to be affine. In this scheme, the key is to show that the Gauss map is constant. The aim of the present paper then is to obtain Bernstein type result for higher codimension by using the geometric properties of the Grassmann manifolds $\mathbf{G}_{n, m}$ and studying the corresponding harmonic Gauss maps into $\mathbf{G}_{n, m}$.

1991 Mathematics Subject Classification. 58E20,53A10.

The first author is supported by the ERC Advanced Grant FP7-267087, the second and third authors are partially supported by NSFC. They are grateful to the Max Planck Institute for Mathematics in the Sciences in Leipzig for its hospitality and continuous support. 
This method was introduced by Hildebrandt-Jost-Widman [12]. The distance function from a fixed point $P_{0} \in \mathbf{G}_{n, m}$ in a geodesic ball of radius $\frac{\sqrt{2}}{4} \pi$ and centered at $P_{0}$ is convex. Using this fact one obtains a strongly subharmonic function on $M$ by composing the distance function with the Gauss map $\gamma$ under the assumption that the image of the Gauss map is contained in a closed subset of the geodesic convex ball. Suppressing some basic technical difficulties in this introduction, an application of the maximum principle should then yield that this subharmonic function is constant, and hence so then is the Gauss map. Using this local convex geometry of Grassmann manifolds and advanced harmonic map regularity theory to overcome the indicated technical difficulties, Hildebrandt-Jost-Widman obtained Bernstein type results for higher dimension and codimension. Somehow, however, while a geodesic ball of radius $\frac{\sqrt{2}}{4} \pi$ is the largest convex ball for $m \geq 2$ (for $m=1$, we can take the ball of the radius $\frac{1}{2} \pi$, that is, a hemisphere), it is not the largest convex set, and therefore the result of [12] is not yet the best possible, and there seems opportunity for improvement. In [15], then, the largest such geodesic convex set was found, and stronger results were obtained. But, as we shall explore here, there is still further opportunity for improvement, via a deeper understanding of the convex geometry of Grassmann manifolds.

Since the geometry of general Grassmann manifolds is not as easy to visualize as the one for the special case $m=1$ where the Grassmannian reduces to the sphere $S^{n}$, let us briefly discuss the situation for the latter. In general, the domain of a strictly convex function cannot contain any closed geodesic, and therefore, a closed hemipshere in $S^{n}$ cannot support a strictly convex function. Thus, no ball with a radius $\geq \frac{\pi}{2}$ can support a strictly convex function. Nevertheless, there exist larger open sets that contain an open hemisphere, but still do not contain any closed geodesic. In fact, on $S^{2}$, we can take the complement $U$ of a semicircle. In [16], we have shown that any compact subset of $U$ supports a strictly convex function. (It is, however, not true that this convex supporting property holds for any open subset of $S^{n}$ containing no closed geodesic.) First of all, this inspires us to look for such convex supporting sets in general Grassmann manifolds. Secondly, the reason why we need convex functions is that the postcomposition of a harmonic map (in our case, the Gauss map of a minimal submanifold) with a convex function is subharmonic so that we can apply the maximum principle. When suitable technical conditions are met (their verification is part of our technical achievements), this tells us that the composition, being subharmonic, is constant. When the function is nontrivial, we can then conclude that the Gauss map itself is constant. Bingo - the minimal submanifold is affine! Now, however, it might happen that the postcomposition of the Gauss map with a function $f$ turns out to be subharmonic without $f$ being convex. Here, the more subtle geometry of the Grassmann manifolds for $m \geq 2$ enters. An intuition about the geometry comes from the following observation. We can rotate the plane $e_{1} \wedge e_{2}$ in $\mathbb{R}^{4}$ either into the plane $e_{1} \wedge e_{3}$ or into the plane $e_{3} \wedge e_{4}$. Each of these rotations yields a geodesic arc in $\mathbf{G}_{2,2}$. However, these two geodesics stop diverging from their neighboring geodesics at different distances $\left(\frac{\sqrt{2}}{4} \pi\right.$ vs. $\left.\frac{\pi}{2}\right)$ (equivalently, the first conjugate point is reached after a distance of $\frac{\sqrt{2}}{2} \pi$ vs. $\left.\pi\right)$, and therefore, a maximal geodesically convex set is larger than a ball 
of radius $\frac{\sqrt{2}}{4} \pi$. While this geometric intuition will be important for the present paper, nevertheless, due to more subtle features of Grassmannian geometry, we can construct suitable functions that are no longer convex, but still give the required subharmonicity on even larger sets than such maximal geodesically convex sets. The function that we use is defined in a geometric manner from the Plücker embedding, or equivalently, in terms of Jordan angles. It is designed to take into account the phenomenon just described, namely that rotations of subspaces of Euclidean space are geometrically different depending on how many independent normal directions are involved. Again, this is a feature particular for $m \geq 2$, not yet apparent on a sphere. In fact, and this is a main point of this paper, we shall find such functions $f$ here, defined on larger subsets of $\mathbf{G}_{n, m}$ than in previous work (as we shall explain in more detail below, these subsets are larger and more natural than the ones identified in our previous work [17]). Therefore, we can obtain a larger possible range of the Gauss map of a minimal submanifold that still implies that it is constant. Thus, we obtain stronger Bernstein type results than previously known. Actually, our approach, being quantitative, also yields estimates for the second fundamental form of minimal submanifolds under suitable geometric conditions.

Of course, there are limits how far one can push Bernstein theorems in higher codimension. For codimension 1, we have Moser's theorem [19] that any entire minimal graph with bounded slope is affine linear (for $n \leq 7$, the bounded slope condition is not needed - this is Simons' theorem [21]). In higher codimensions, the situation is not so good. After all, there is the important example of Lawson-Osserman [18] of a nontrivial minimal graph (to be analyzed in detail in the Appendix of the present paper) of bounded slope that sets a limit for how far one can go. The ultimate aim then is to narrow the gap between the range of Bernstein theorems and the counterexamples as far as possible. Our paper is a step in this direction. We do not know yet whether one can still go further, or whether there exist even more striking examples than the one of [18].

One more remark: Most of what we do in this paper holds for submanifolds of parallel mean curvature, and not only for those of vanishing mean curvature, the minimal ones. Since, however, according to mathematical tradition, the minimal submanifolds are the most interesting ones, in this introduction, we mostly restrict ourselves to discuss those minimal ones in place of the more general ones of parallel mean curvature.

Let us now describe the results in more precise terms. The Grassmann manifold $\mathbf{G}_{n, m}$ can be imbedded into Euclidean space by the Plücker imbedding. This simply means that we consider an oriented $n$-plane in $\mathbb{R}^{n+m}$ as an element of $\Lambda^{n}\left(\mathbb{R}^{n+m}\right)$. In this space, we also have a scalar product. We then can introduce suitable functions, called $w$-functions and $v$-functions, on $\mathbf{G}_{n, m}$. The $w$-function is simply given by the above scalar product with some fixed reference $n$-plane, and $v$ is the inverse of $w$. By precomposition with the Gauss map, we then get corresponding functions on $M$. The region of convexity for the $v$-function is the same as for the corresponding distance function [26], but in our previous work [17] we have already found that there is a larger region in $\mathbf{G}_{n, m}$ on which the $v$-function is no longer convex, but 
where its composition with the Gauss map is still strongly subharmonic by rather delicate estimates. We then obtain the following theorem

Theorem 1.1. Let $z^{\alpha}=f^{\alpha}\left(x^{1}, \cdots, x^{n}\right), \alpha=1, \cdots, m$, be smooth functions defined everywhere in $\mathbb{R}^{n}(n \geq 3, m \geq 2)$. Suppose their graph $M=(x, f(x))$ is a submanifold with parallel mean curvature in $\mathbb{R}^{n+m}$. Suppose that there exists a number $\beta_{0}<3$ such that

$$
\Delta_{f}=\left[\operatorname{det}\left(\delta_{i j}+\sum_{\alpha} \frac{\partial f^{\alpha}}{\partial x^{i}} \frac{\partial f^{\alpha}}{\partial x^{j}}\right)\right]^{\frac{1}{2}} \leq \beta_{0} .
$$

Then $f^{1}, \cdots, f^{m}$ has to be affine linear, i.e., it represents an affine $n$-plane.

In our previous work (see [15, 17, 25, 26]) the image under the Gauss map for a submanifold in Euclidean space is contained in an $(n \times m)$-matrix chart for a Grassmann manifold. There is, however, still further room for improvement, and here, we consider a more general situation. Recall that a Grassmann manifold can be viewed as a minimal submanifold in the Euclidean sphere via the Plücker imbedding. This leads us to employ a technique from our previous work [16] for the case of codimension $m=1$, where the convex geometry of the Euclidean sphere has been thoroughly investigated.

We shall introduce new notions of $S$-orthogonality and of $S$-maps on a Grassmann manifold. Let $P, Q \in \mathbf{G}_{n, m}$ be $S$-orthogonal to each other. This means that their intersection is of dimension $n-1$ and one is obtained from the other by rotating a single tangent vector into a normal direction by an angle of $\frac{\pi}{2}$. Using the $w$-function we define an $S$-map $\mathscr{S}: \mathbf{G}_{n, m} \rightarrow \overline{\mathbb{D}}$ relative to $P$ and $Q$. This is a map onto the closed unit disk.

Define

$$
\mathbb{W}_{c}:=\mathscr{S}^{-1}\left(\overline{\mathbb{D}} \backslash\left(\overline{\mathbb{D}}_{c} \cup\{(a, 0): a \leq 0\}\right)\right),
$$

with $\overline{\mathbb{D}}_{c}=\left\{\left(x_{1}, x_{2}\right) \in \mathbb{R}^{2}: x_{1}^{2}+x_{2}^{2} \leq c^{2}\right\}$.

Now, for our method to apply, the image of our Gauss map can be any compact subset of $\mathbb{W}_{\frac{1}{3}}$. This is a global region in $\mathbf{G}_{n, m}$ that is not contained in any matrix coordinate chart. More precisely, even when the Gauss image is somewhat larger, we still can find subharmonic functions on our submanifold by combining some tricks in our previous work in [16] and [17]. In this way, we obtain the following Bernstein type theorem.

Theorem 1.2. Let $z^{\alpha}=f^{\alpha}\left(x^{1}, \cdots, x^{n}\right), \alpha=1, \cdots, m$, be smooth functions defined everywhere in $\mathbb{R}^{n}(n \geq 3, m \geq 2)$, such that their graph $M=(x, f(x))$ is a submanifold with parallel mean curvature in $\mathbb{R}^{n+m}$. Suppose that there exist $\beta_{0}<+\infty$ and $\beta_{1}<3$, such that

$$
\Delta_{f}:=\left[\operatorname{det}\left(\delta_{i j}+\sum_{\alpha} \frac{\partial f^{\alpha}}{\partial x^{i}} \frac{\partial f^{\alpha}}{\partial x^{j}}\right)\right]^{\frac{1}{2}} \leq \beta_{0} .
$$


and for certain $\alpha$ and $i$

$$
\Delta_{f} \leq \beta_{1}\left(1+\left(\frac{\partial f^{\alpha}}{\partial x^{i}}\right)^{2}\right)^{\frac{1}{2}}
$$

Then $f^{1}, \cdots, f^{m}$ has to be affine linear (representing an affine $n$-plane).

In fact, we can prove more general results, see Theorem 6.1 in $\S 6$, but the preceding statement perhaps best highlights the main achievement of this paper.

In the Appendix we see that for the Lawson-Osserman cone the $v$-function is the constant 9. The Jordan angles between the Gauss image of this cone and the coordinate $n$ - plane are constants. The largest value for a Jordan angle permitted by

the refined term $\left(1+\frac{\partial f^{2}}{\partial x^{1}}\right)^{\frac{1}{2}}$ in the above Theorem is $\sqrt{6}$. Theorem 1.2 is a substantial improvement of the previous results which only could reach smaller values.

The paper is organized as follows. We will describe in $\S 2$ that the basic geometry of Grassmann manifolds and define S-orthogonality and S-maps and show their properties. $\S 3$ will be devoted to the computation of $\Delta \log w$. For the general case the results come from [26]. We also compute it for minimal submanifolds with rank of the Gauss map less than or equal 2. We obtain a formula more general than that in [6]. In $\S 4$ we will construct subharmonic functions $F$ on our submanifolds by using the techniques in [16]. The level sets of $F$ coincide with those of $\log w$. We first prove an important transition lemma (Lemma 4.1). Then, we can estimate Hess $F$ in terms of Hess $\log w$, which is already computed in the previous section. Once we have subharmonic functions, the extrinsic rigidity results for compact minimal submanifolds in the sphere (Theorem 4.2) follows immediately. Using strongly subharmonic functions we can also study complete submanifolds in Euclidean space by using harmonic map regularity theory, as Hildebrandt-Jost-Widman in [12]. In $\S 5$ we will obtain curvature estimates (Lemma 5.3). In $\S 6$ the iteration method will be used and the quantitatively controlled Gauss image shrinking Lemmas 6.2 and 6.3 will be obtained. Finally, in this section we prove our main results, Theorem 6.1 and Theorem 6.2 of which Theorem 1.2 is a direct corollary. Finally, in an Appendix, we provide computations for the Lawson-Osserman cone and related coassociated 4-manifolds in $\mathbb{R}^{7}$.

\section{Geometry of Grassmann manifolds}

Let $\mathbf{G}_{n, m}$ be the Grassmann manifold consisting of the oriented linear $n$-subspaces in $(n+m)$-Euclidean space $\mathbb{R}^{n+m}$. The canonical Riemannian structure on $\mathbf{G}_{n, m}$ makes it a natural generalization of the Euclidean sphere. $\mathbf{G}_{n, m}=S O(n+m) / S O(n) \times$ $S O(m)$ is an irreducible symmetric space of compact type.

For every $P \in \mathbf{G}_{n, m}$, we choose an oriented basis $\left\{u_{1}, \cdots, u_{n}\right\}$ of $P$, and let

$$
\psi(P):=u_{1} \wedge \cdots \wedge u_{n} \in \Lambda^{n}\left(\mathbb{R}^{n+m}\right) .
$$


A different basis for $P$ shall give a different exterior product, but the two products differ only by a positive scalar; $\psi(P)$ is called the Plücker coordinate of $P$, which is a homogenous coordinate.

Via the Plücker embedding, $\mathbf{G}_{n, m}$ can be viewed as a submanifold of some Euclidean space $\mathbb{R}^{N}\left(N=C_{n+m}^{n}\right)$. The restriction of the Euclidean inner product is denoted by $w: \mathbf{G}_{n, m} \times \mathbf{G}_{n, m} \rightarrow \mathbb{R}$

$$
w(P, Q)=\frac{\langle\psi(P), \psi(Q)\rangle}{\langle\psi(P), \psi(P)\rangle^{\frac{1}{2}}\langle\psi(Q), \psi(Q)\rangle^{\frac{1}{2}}} .
$$

If $\left\{e_{1}, \cdots, e_{n}\right\}$ is an oriented orthonormal basis of $P$ and $\left\{f_{1}, \cdots, f_{n}\right\}$ is an oriented orthonormal basis of $Q$, then

$$
w(P, Q)=\left\langle e_{1} \wedge \cdots \wedge e_{n}, f_{1} \wedge \cdots \wedge f_{n}\right\rangle=\operatorname{det} W
$$

with the $W$-matrix $W=\left(\left\langle e_{i}, f_{j}\right\rangle\right)$. It is well-known that

$$
W^{T} W=O^{T} \Lambda O
$$

with an orthogonal matrix $O$ and

$$
\Lambda=\left(\begin{array}{ccc}
\mu_{1}^{2} & & \\
& \ddots & \\
& & \mu_{n}^{2}
\end{array}\right) .
$$

Here each $0 \leq \mu_{i}^{2} \leq 1$. Putting $p:=\min \{m, n\}$, then at most $p$ elements in $\left\{\mu_{1}^{2}, \cdots, \mu_{n}^{2}\right\}$ are not equal to 1 . Without loss of generality, we can assume $\mu_{i}^{2}=1$ whenever $i>p$. We also note that the $\mu_{i}^{2}$ can be expressed as

$$
\mu_{i}^{2}=\frac{1}{1+\lambda_{i}^{2}}
$$

with $\lambda_{i} \in[0,+\infty)$.

The Jordan angles between $P$ and $Q$ are critical values of the angle $\theta$ between a nonzero vector $u$ in $P$ and its orthogonal projection $u^{*}$ in $Q$ as $u$ runs through $P$. Let $\theta_{i}$ be a Jordan angle between $P$ and $Q$ determined by a unit vector $e_{i}$ and its projection $e_{i}^{*}$ in $Q$, we call $e_{i}$ an angle direction of $P$ relative to $Q$, and the 2-plane spanned by $e_{i}$ and $e_{i}^{*}$ an angle 2-plane between $P$ and $Q$ (see [22]). A direct calculation shows there are $n$ Jordan angles $\theta_{1}, \cdots, \theta_{n}$, with $\theta_{p+1}=\cdots=\theta_{n}=0$ and

$$
\theta_{i}=\arccos \left(\mu_{i}\right) \quad 1 \leq i \leq p
$$

Thus

$$
|w|=\left(\operatorname{det}\left(W^{T} W\right)\right)^{\frac{1}{2}}=\operatorname{det}(\Lambda)^{\frac{1}{2}}=\prod_{i=1}^{n} \cos \theta_{i}
$$

and (2.3) becomes

$$
\lambda_{i}=\tan \theta_{i}
$$


If $w(P, Q)>0$, arrange all the Jordan angles between $P$ and $Q$ as

$$
\frac{\pi}{2}>\theta_{1} \geq \theta_{2} \geq \cdots \geq \theta_{r}>\theta_{r+1}=\cdots=\theta_{n}=0
$$

with $0 \leq r \leq p$. (If $\theta_{i}=\frac{\pi}{2}$ for some $i$, then (2.4) implies $w(P, Q)=0$, which contradicts $w(P, Q)>0$.) Then one can find an orthonormal basis $\left\{e_{1}, \cdots, e_{n+m}\right\}$ of $\mathbb{R}^{n+m}$, such that $P$ is spanned by $\left\{e_{1}, \cdots, e_{n}\right\}$, which are angle directions of $P$ relative to $Q$, and $\left\{e_{i} \wedge e_{n+i}: 1 \leq i \leq r\right\}$ are angle 2-planes between $P$ and $Q$. Denote

$$
f_{i}:=\left\{\begin{array}{cc}
\cos \theta_{i} e_{i}+\sin \theta_{i} e_{n+i} & 1 \leq i \leq r \\
e_{i} & r+1 \leq i \leq n
\end{array}\right.
$$

and

$$
f_{n+\alpha}:=\left\{\begin{array}{cc}
-\sin \theta_{\alpha} e_{\alpha}+\cos \theta_{\alpha} e_{n+\alpha} & 1 \leq \alpha \leq r \\
e_{n+\alpha} & r+1 \leq \alpha \leq m .
\end{array}\right.
$$

then $\left\{f_{1}, \cdots, f_{n+m}\right\}$ is also an orthonormal basis of $\mathbb{R}^{n+m}$, and $f_{1} \wedge \cdots \wedge f_{n}$ is a Plücker coordinate of $Q$.

The distance between $P$ and $Q$ is defined by

$$
d(P, Q)=\sqrt{\sum \theta_{i}^{2}}
$$

It is a natural generalization of the canonical distance of Euclidean spheres.

Now we fix $P_{0} \in \mathbf{G}_{n, m}$. We represent it by $n$ vectors $\epsilon_{i}$, which are complemented by $m$ vectors $\epsilon_{n+\alpha}$. Denote

$$
\mathbb{U}:=\left\{P \in \mathbf{G}_{n, m}: w\left(P, P_{0}\right)>0\right\} .
$$

We can span an arbitrary $P \in \mathbb{U}$ by $n$ vectors $e_{i}$ :

$$
e_{i}=\epsilon_{i}+Z_{i \alpha} \epsilon_{n+\alpha}
$$

Here $Z=\left(Z_{i \alpha}\right)$ could be regarded as the $(n \times m)$-matrix coordinate of $P$. The canonical Riemannian metric in $\mathbb{U}$ can be described as

$$
d s^{2}=\operatorname{tr}\left(\left(I_{n}+Z Z^{T}\right)^{-1} d Z\left(I_{m}+Z^{T} Z\right)^{-1} d Z^{T}\right)
$$

where $I_{m}\left(\right.$ res. $\left.I_{n}\right)$ denotes the $(m \times m)$-identity $($ res. $n \times n)$ matrix. It is shown that (2.11) can be derived from (2.8) in [23].

Here and in the sequel, we use the summation convention and agree on the ranges of indices:

$$
1 \leq \alpha, \beta \leq m, 1 \leq i, j, k \leq n
$$

We shall now introduce the new concepts of $S$-orthogonal and $S$-map, which will play a crucial role for our investigations.

Proposition 2.1. For any $P, Q \in \mathbf{G}_{n, m}$, the following statements are equivalent:

(a) $\operatorname{dim}(P \cap Q)=n-1$ and $w(P, Q)=0$;

(b) $\operatorname{dim}(P+Q)=n+1$ and $w(P, Q)=0$, where $P+Q=\{u+v: u \in P, v \in Q\}$;

(c) There exists a single Jordan angle between $P$ and $Q$ being $\frac{\pi}{2}$, and the other $(n-1)$ Jordan angles are all 0; 
(d) $Q$ is the nearest point to $P$ in $\partial \mathbb{U}$, where $\mathbb{U}$ is the $(n \times m)$-matrix coordinate chart centered at $P$;

(e) There exists an orthonormal basis $\left\{e_{i}, e_{n+\alpha}\right\}$ of $\mathbb{R}^{n+m}$, such that the Plücker coordinates of $P$ and $Q$ are $e_{1} \wedge e_{2} \wedge \cdots \wedge e_{n}$ and $e_{n+1} \wedge e_{2} \wedge \cdots \wedge e_{n}$, respectively.

Proof. $(\mathrm{a}) \Longleftrightarrow(\mathrm{b})$ follows from $\operatorname{dim}(P+Q)=\operatorname{dim} P+\operatorname{dim} Q-\operatorname{dim}(P \cap Q)$.

$(\mathrm{a}) \Longleftrightarrow(\mathrm{c})$ is an immediate corollary of the definition of Jordan angles and (2.4).

$\partial \mathbb{U}$ consists of all $S \in \mathbf{G}_{n, m}$ satisfying $w(P, S)=0$; it follows from (2.4) that at least 1 Jordan angle between $S$ and $P$ is $\frac{\pi}{2}$; hence one can obtain

$$
d(P, S) \geq \frac{\pi}{2} \quad \text { for all } S \in \partial \mathbb{U}
$$

from (2.8), and the equality holds if and only if the other $(n-1)$ Jordan angles all vanish. Hence $(\mathrm{c}) \Longleftrightarrow(\mathrm{d})$.

$(\mathrm{e}) \Longrightarrow(\mathrm{a})$ is trivial. To prove $(\mathrm{a}) \Longrightarrow(\mathrm{e})$, it suffices to choose $\left\{e_{2}, \cdots, e_{n}\right\}$ as an orthonormal basis of $P \cap Q$, and put $e_{1}$ (or $e_{n+1}$ ) to be the unit vector in $P$ (or $Q$ ) that is orthogonal to $P \cap Q$.

Definition 2.1. Two points $P$ and $Q$ in a Grassmann manifold are called $S$ orthogonal, if they satisfy one of the properties in Proposition 2.1.

Remark 2.1. There is only one Jordan angle between any two points in the sphere $S^{n}$. If it equals $\frac{\pi}{2}$, they are orthogonal each other. The notion of $S$-orthogonal is a natural generalization of orthogonality in $S^{n}$.

Let $P, Q \in \mathbf{G}_{n, m}$ be S-orthogonal. By Proposition 2.1, there exists an orthonormal basis $\left\{e_{i}, e_{n+\alpha}\right\}$ of $\mathbb{R}^{n+m}$, such that $\psi(P)=e_{1} \wedge e_{2} \wedge \cdots \wedge e_{n}$ and $\psi(Q)=e_{n+1} \wedge e_{2} \wedge$ $\cdots \wedge e_{n}$. Let $\gamma: \mathbb{R} /(2 \pi \mathbb{Z}) \rightarrow \mathbf{G}_{n, m}$ be a closed curve, such that $\gamma(t)$ is spanned by $\left\{\cos t e_{1}+\sin t e_{n+1}, e_{2}, \cdots, e_{n}\right\}$. Then whenever $|t-s|<\frac{\pi}{2}$, there exists one and only one Jordan angle between $\gamma(t)$ and $\gamma(s)$ being $|t-s|$, and the other Jordan angles are all 0; hence by (2.8),

$$
d(\gamma(t), \gamma(s))=|t-s| \quad \text { whenever }|t-s|<\frac{\pi}{2} .
$$

It implies $\gamma$ is the closed geodesic that is extended from the minimal geodesic between $P$ and $Q$. In the following, we write $P_{t}=\gamma(t)$; in particular, $P_{0}=P$ and $P_{\frac{\pi}{2}}=Q$.

The $S$-map $\mathscr{S}: \mathbf{G}_{n, m} \rightarrow \mathbb{R}^{2}$ is defined by

$$
S \mapsto\left(w\left(S, P_{0}\right), w\left(S, P_{\frac{\pi}{2}}\right)\right) .
$$

If there exists a nonzero vector in $S$ orthogonal to $P_{0} \cap P_{\frac{\pi}{2}}$, then $w\left(S, P_{0}\right)=$ $w\left(S, P_{\frac{\pi}{2}}\right)=0$, and hence $\mathscr{S}(S)=0$. Otherwise, the orthogonal projection $p$ from $P_{0} \cap P_{\frac{\pi}{2}}^{2}$ to $S$ has rank $n-1$, which enables us to find a unit vector $f_{1}$ in $S$ which is orthogonal to the $(n-1)$-dimensional projective image $p\left(P_{0} \cap P_{\frac{\pi}{2}}\right)$. Certainly, one 
can get

$$
\begin{gathered}
w\left(S, P_{0}\right)=\left\langle f_{1}, e_{1}\right\rangle w\left(p\left(P_{0} \cap P_{\frac{\pi}{2}}\right), P_{0} \cap P_{\frac{\pi}{2}}\right), \\
w\left(S, P_{\frac{\pi}{2}}\right)=\left\langle f_{1}, e_{n+1}\right\rangle w\left(p\left(P_{0} \cap P_{\frac{\pi}{2}}\right), P_{0} \cap P_{\frac{\pi}{2}}\right) .
\end{gathered}
$$

It follows that

$$
w^{2}\left(S, P_{0}\right)+w^{2}\left(S, P_{\frac{\pi}{2}}\right) \leq\left\langle f_{1}, e_{1}\right\rangle^{2}+\left\langle f_{1}, e_{n+1}\right\rangle^{2} \leq 1
$$

and the equality holds if and only if $p\left(P_{0} \cap P_{\frac{\pi}{2}}\right)=P_{0} \cap P_{\frac{\pi}{2}}$ and $f_{1} \in \operatorname{span}\left\{e_{1}, e_{n+1}\right\}$; i.e. $S=P_{t}$ for some $t \in[-\pi, \pi)$. Hence $\mathscr{S}$ is a smooth map onto the closed unit 2-disk $\overline{\mathbb{D}}$. Although $\mathscr{S}$ is not one-to-one, any point on $\partial \overline{\mathbb{D}}$ has one and only one preimage. For simplicity we write

$$
x_{1}=w\left(\cdot, P_{0}\right), \quad x_{2}=w\left(\cdot, P_{\frac{\pi}{2}}\right) .
$$

Then from (2.13) one can obtain

$$
\begin{aligned}
\left(\cos t x_{1}+\sin t x_{2}\right)(S) & =\cos t w\left(S, P_{0}\right)+\sin t w\left(S, P_{\frac{\pi}{2}}\right) \\
& =\left\langle f_{1}, \cos t e_{1}+\sin t e_{n+1}\right\rangle w\left(p\left(P_{0} \cap P_{\frac{\pi}{2}}\right), P_{0} \cap P_{\frac{\pi}{2}}\right) \\
& =w\left(S, P_{t}\right) .
\end{aligned}
$$

\section{LAPLACIAN OF $\log w$}

Let $M^{n} \rightarrow \bar{M}^{n+m}$ be an isometric immersion with second fundamental form $B$, which can be viewed as a cross-section of the vector bundle $\operatorname{Hom}\left(\odot^{2} T M, N M\right)$ over $M$, where $T M$ and $N M$ denote the tangent bundle and the normal bundle along $M$, respectively. The connection on $T M$ and $N M$ (denoted by $\nabla$ ) can be induced naturally from the Levi-Civita connection on $\bar{M}$ (denoted by $\bar{\nabla}$ ). We define the mean curvature $H$ as the trace of the second fundamental form. It is a normal vector field on $M$ in $\bar{M}$. If $\nabla H=0$, we say $M$ has parallel mean curvature; moreover if $H$ vanishes on $M$ everywhere, it is called a minimal submanifold.

The second fundamental form, the curvature tensor of the submanifold (denoted by $R$ ), the curvature tensor of the normal bundle (denoted by $R^{N}$ ) and that of the ambient manifold (denoted by $\bar{R}$ ) satisfy the Gauss equations, the Codazzi equations and the Ricci equations (see [23] for details, for example). Here and in the sequel, $\left\{e_{i}\right\}$ denotes a local orthonormal tangent frame field and $\left\{\nu_{\alpha}\right\}$ is a local orthonormal normal frame field of $M$;

$$
h_{\alpha, i j}:=\left\langle\left\langle B\left(e_{i}, e_{j}\right), \nu_{\alpha}\right\rangle\right.
$$

are the coefficients of the second fundamental form $B$ of $M$ in $\bar{M}$.

Now we consider a submanifold $M^{n}$ in Euclidean space $\mathbb{R}^{n+m}$ with parallel mean curvature.

Let 0 be the origin of $\mathbb{R}^{n+m}, S O(n+m)$ be the Lie group consisting of all orthonormal frames $\left(0 ; e_{i}, \nu_{\alpha}\right) . T F=\left\{\left(x ; e_{1}, \cdots, e_{n}\right): x \in M, e_{i} \in T_{p} M,\left\langle e_{i}, e_{j}\right\rangle=\right.$ $\left.\delta_{i j}\right\}$ be the principle bundle of orthonormal tangent frames over $M$, and $N F=$ 
$\left\{\left(x ; \nu_{1}, \cdots, \nu_{m}\right): x \in M, \nu_{\alpha} \in N_{x} M\right\}$ be the principle bundle of orthonormal normal frames over $M$. Then $\bar{\pi}: T F \oplus N F \rightarrow M$ is the projection with fiber $S O(n) \times S O(m)$.

The Gauss map $\gamma: M \rightarrow \mathbf{G}_{n, m}$ is defined by

$$
\gamma(x)=T_{x} M \in \mathbf{G}_{n, m}
$$

via the parallel translation in $\mathbb{R}^{n+m}$ for every $p \in M$. Then the following diagram commutes

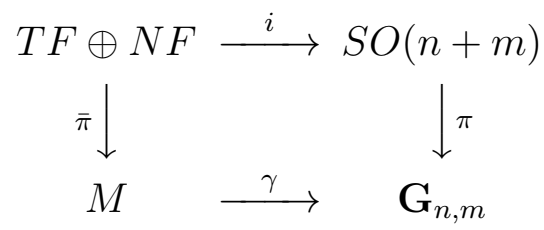

where $i$ denotes the inclusion map and $\pi: S O(n+m) \rightarrow \mathbf{G}_{n, m}$ is defined by

$$
\left(0 ; e_{i}, \nu_{\alpha}\right) \mapsto e_{1} \wedge \cdots \wedge e_{n}
$$

From the above diagram we know that the energy density of the Gauss map (see [23] Chap.3, §3.1)

$$
e(\gamma)=\frac{1}{2}\left\langle\gamma_{*} e_{i}, \gamma_{*} e_{i}\right\rangle=\frac{1}{2}|B|^{2} .
$$

Ruh-Vilms proved that the mean curvature vector of $M$ is parallel if and only if its Gauss map is a harmonic map [20].

We define

$$
w:=w\left(\cdot, P_{0}\right) \circ \gamma
$$

Let $\left\{\varepsilon_{i}\right\}$ be an orthonormal basis of $P_{0}$, then $w=\left\langle e_{1} \wedge \cdots \wedge e_{n}, \varepsilon_{1} \wedge \cdots \wedge \varepsilon_{n}\right\rangle$ at every point $x \in M$. From the Codazzi equations, we shall now get basic formulas for the function $w$.

Lemma 3.1. [6] [24] If $M$ is a submanifold in $\mathbb{R}^{n+m}$, then

$$
\nabla_{e_{i}} w=h_{\alpha, i j}\left\langle e_{j \alpha}, \varepsilon_{1} \wedge \cdots \wedge \varepsilon_{n}\right\rangle
$$

with

$$
e_{j \alpha}=e_{1} \wedge \cdots \wedge \nu_{\alpha} \wedge \cdots \wedge e_{n}
$$

that is obtained by replacing $e_{j}$ by $\nu_{\alpha}$ in $e_{1} \wedge \cdots \wedge e_{n}$. Moreover if $M$ has parallel mean curvature, then

$$
\Delta w=-|B|^{2} w+\sum_{i} \sum_{\alpha \neq \beta, j \neq k} h_{\alpha, i j} h_{\beta, i k}\left\langle e_{j \alpha, k \beta}, \varepsilon_{1} \wedge \cdots \wedge \varepsilon_{n}\right\rangle .
$$

with

$$
e_{j \alpha, k \beta}=e_{1} \wedge \cdots \wedge \nu_{\alpha} \wedge \cdots \wedge \nu_{\beta} \wedge \cdots \wedge e_{n}
$$

that is obtained by replacing $e_{j}$ by $\nu_{\alpha}$ and $e_{k}$ by $\nu_{\beta}$ in $e_{1} \wedge \cdots \wedge e_{n}$, respectively. 
Now we compute $\Delta \log w$ under the additional assumption that $H=0$ and the rank of the Gauss map $\gamma$ is at most 2. Without loss of generality, one can assume $h_{i j}^{\alpha}=0$ whenever $i \geq 3$ or $j \geq 3$. Then, we need to calculate $\left\langle e_{1 \alpha, 2 \beta}, \varepsilon_{1} \wedge \cdots \wedge \varepsilon_{n}\right\rangle$. The following identity shall play an important role.

Lemma 3.2. Fix $A=\varepsilon_{1} \wedge \cdots \wedge \varepsilon_{n}$, then for any distinct indices $\alpha, \beta$,

$$
\left\langle e_{1} \wedge \cdots \wedge e_{n}, A\right\rangle\left\langle e_{1 \alpha, 2 \beta}, A\right\rangle-\left\langle e_{1 \alpha}, A\right\rangle\left\langle e_{2 \beta}, A\right\rangle+\left\langle e_{1 \beta}, A\right\rangle\left\langle e_{2 \alpha}, A\right\rangle=0
$$

Proof. Let $Q \in \mathbf{G}_{n-2, m+2}$ spanned by $\left\{e_{i}: 3 \leq i \leq n\right\}$. If there is a nonzero vector in $Q$ which is orthogonal to the $n$-dimensional space $P_{0}$ spanned by $\left\{\varepsilon_{i}\right\}$, then by the definition of the inner product on $\Lambda^{n}\left(\mathbb{R}^{n+m}\right)$, all the terms on the left hand side of (3.6) equal 0 and (3.6) trivially holds true.

Otherwise the orthogonal projection $p: Q \rightarrow P_{0}$ has rank $n-2$. Without loss of generality we assume $p(Q)$ is spanned by $\left\{\varepsilon_{i}: 3 \leq i \leq n\right\}$; the Jordan angles between $Q$ and $p(Q)$ are denoted by $\theta_{3}, \cdots, \theta_{n}$; and $\theta_{i}$ is determined by $e_{i}$ and $\varepsilon_{i}$. Hence $\left\langle e_{i}, \varepsilon_{j}\right\rangle=\cos \theta_{i} \delta_{i j}$ whenever $i \geq 3$, and moreover

$$
\begin{aligned}
\left\langle e_{1} \wedge \cdots \wedge e_{n}, A\right\rangle & =\left|\begin{array}{cccc}
\left(e_{1}, \varepsilon_{1}\right) & \left(e_{1}, \varepsilon_{2}\right) & * \\
\left(e_{2}, \varepsilon_{1}\right) & \left(e_{2}, \varepsilon_{2}\right) & & \\
0 & \cos \theta_{3} & & \\
0 & \ddots & \cos \theta_{n}
\end{array}\right| \\
& =\left\langle e_{1} \wedge e_{2}, \varepsilon_{1} \wedge \varepsilon_{2}\right\rangle \prod_{i=3}^{n} \cos \theta_{i}
\end{aligned}
$$

Similarly,

$$
\left\langle e_{1 \alpha, 2 \beta}, A\right\rangle=\left\langle\nu_{\alpha} \wedge \nu_{\beta}, \varepsilon_{1} \wedge \varepsilon_{2}\right\rangle \prod_{i=3}^{n} \cos \theta_{i}
$$

and

$$
\begin{aligned}
& \left\langle e_{1 \gamma}, A\right\rangle=\left\langle\nu_{\gamma} \wedge e_{2}, \varepsilon_{1} \wedge \varepsilon_{2}\right\rangle \prod_{i=3}^{n} \cos \theta_{i} \\
& \left\langle e_{2 \gamma}, A\right\rangle=\left\langle e_{1} \wedge \nu_{\gamma}, \varepsilon_{1} \wedge \varepsilon_{2}\right\rangle \prod_{i=3}^{n} \cos \theta_{i}
\end{aligned}
$$


for $\gamma=\alpha$ or $\beta$. A direct calculation shows

$$
\begin{aligned}
& \left\langle e_{1} \wedge e_{2}, \varepsilon_{1} \wedge \varepsilon_{2}\right\rangle\left\langle\nu_{\alpha} \wedge \nu_{\beta}, \varepsilon_{1} \wedge \varepsilon_{2}\right\rangle-\left\langle\nu_{\alpha} \wedge e_{2}, \varepsilon_{1} \wedge \varepsilon_{2}\right\rangle\left\langle e_{1} \wedge \nu_{\beta}, \varepsilon_{1} \wedge \varepsilon_{2}\right\rangle \\
& +\left\langle\nu_{\beta} \wedge e_{2}, \varepsilon_{1} \wedge \varepsilon_{2}\right\rangle\left\langle e_{1} \wedge \nu_{\alpha}, \varepsilon_{1} \wedge \varepsilon_{2}\right\rangle \\
= & +\left(e_{1}, \varepsilon_{1}\right)\left(e_{2}, \varepsilon_{2}\right)\left(\nu_{\alpha}, \varepsilon_{1}\right)\left(\nu_{\beta}, \varepsilon_{2}\right)+\left(e_{2}, \varepsilon_{1}\right)\left(e_{1}, \varepsilon_{2}\right)\left(\nu_{\beta}, \varepsilon_{1}\right)\left(\nu_{\alpha}, \varepsilon_{2}\right) \\
& -\left(e_{1}, \varepsilon_{1}\right)\left(e_{2}, \varepsilon_{2}\right)\left(\nu_{\beta}, \varepsilon_{1}\right)\left(\nu_{\alpha}, \varepsilon_{2}\right)-\left(e_{2}, \varepsilon_{1}\right)\left(e_{1}, \varepsilon_{2}\right)\left(\nu_{\alpha}, \varepsilon_{1}\right)\left(\nu_{\beta}, \varepsilon_{2}\right) \\
& -\left(e_{1}, \varepsilon_{1}\right)\left(e_{2}, \varepsilon_{2}\right)\left(\nu_{\alpha}, \varepsilon_{1}\right)\left(\nu_{\beta}, \varepsilon_{2}\right)-\left(e_{2}, \varepsilon_{1}\right)\left(e_{1}, \varepsilon_{2}\right)\left(\nu_{\beta}, \varepsilon_{1}\right)\left(\nu_{\alpha}, \varepsilon_{2}\right) \\
& +\left(e_{1}, \varepsilon_{2}\right)\left(e_{2}, \varepsilon_{2}\right)\left(\nu_{\alpha}, \varepsilon_{1}\right)\left(\nu_{\beta}, \varepsilon_{1}\right)+\left(e_{1}, \varepsilon_{1}\right)\left(e_{2}, \varepsilon_{1}\right)\left(\nu_{\alpha}, \varepsilon_{2}\right)\left(\nu_{\beta}, \varepsilon_{2}\right) \\
& +\left(e_{1}, \varepsilon_{1}\right)\left(e_{2}, \varepsilon_{2}\right)\left(\nu_{\beta}, \varepsilon_{1}\right)\left(\nu_{\alpha}, \varepsilon_{2}\right)+\left(e_{2}, \varepsilon_{1}\right)\left(e_{1}, \varepsilon_{2}\right)\left(\nu_{\alpha}, \varepsilon_{1}\right)\left(\nu_{\beta}, \varepsilon_{2}\right) \\
& -\left(e_{1}, \varepsilon_{2}\right)\left(e_{2}, \varepsilon_{2}\right)\left(\nu_{\alpha}, \varepsilon_{1}\right)\left(\nu_{\beta}, \varepsilon_{1}\right)-\left(e_{1}, \varepsilon_{1}\right)\left(e_{2}, \varepsilon_{1}\right)\left(\nu_{\alpha}, \varepsilon_{2}\right)\left(\nu_{\beta}, \varepsilon_{2}\right) \\
= & 0 .
\end{aligned}
$$

From (3.7)-(3.11), (3.6) immediately follows.

$$
H=0 \text { implies } 0=h_{\alpha, i i}=h_{\alpha, 11}+h_{\alpha, 22} \text { for every } 1 \leq \alpha \leq m \text {, thus }
$$

$$
\begin{aligned}
h_{\alpha, i 1} h_{\beta, i 2} & =h_{\alpha, 11} h_{\beta, 12}+h_{\alpha, 21} h_{\beta, 22} \\
& =-h_{\alpha, 22} h_{\beta, 12}-h_{\alpha, 21} h_{\beta, 11}=-h_{\alpha, i 2} h_{\beta, i 1}
\end{aligned}
$$

and in particular $h_{\alpha, i 1} h_{\alpha, i 2}=0$. Therefore (3.4) can be rewritten as

$$
\begin{aligned}
\Delta w & =-|B|^{2} w+\sum_{\alpha \neq \beta} h_{\alpha, i 1} h_{\beta, i 2}\left\langle e_{1 \alpha, 2 \beta}, A\right\rangle+\sum_{\alpha \neq \beta} h_{\alpha, i 2} h_{\beta, i 1}\left\langle e_{1 \beta, 2 \alpha}, A\right\rangle \\
& =-|B|^{2} w+2 \sum_{\alpha, \beta} h_{\alpha, i 1} h_{\beta, i 2}\left\langle e_{1 \alpha, 2 \beta}, A\right\rangle .
\end{aligned}
$$

By (3.2),

$$
|\nabla w|^{2}=\sum_{i}\left|\nabla_{e_{i}} w\right|^{2}=h_{\alpha, i j} h_{\beta, i k}\left\langle e_{j \alpha}, A\right\rangle\left\langle e_{k \beta}, A\right\rangle .
$$

With the aid of (3.6) and (3.12) one can get

$$
\begin{aligned}
w \Delta w-|\nabla w|^{2}= & -|B|^{2} w^{2}+2 h_{\alpha, i 1} h_{\beta, i 2}\left\langle e_{1} \wedge \cdots \wedge e_{n}, A\right\rangle\left\langle e_{1 \alpha, 2 \beta}, A\right\rangle-h_{\alpha, i j} h_{\beta, i k}\left\langle e_{j \alpha}, A\right\rangle\left\langle e_{k \beta}, A\right\rangle \\
= & -|B|^{2} w^{2}+2 h_{\alpha, i 1} h_{\beta, i 2}\left(\left\langle e_{1 \alpha}, A\right\rangle\left\langle e_{2 \beta}, A\right\rangle-\left\langle e_{1 \beta}, A\right\rangle\left\langle e_{2 \alpha}, A\right\rangle\right) \\
& -h_{\alpha, i j} h_{\beta, i k}\left\langle e_{j \alpha}, A\right\rangle\left\langle e_{k \beta}, A\right\rangle \\
= & -|B|^{2} w^{2}+2 h_{\alpha, i 1} h_{\beta, i 2}\left\langle e_{1 \alpha}, A\right\rangle\left\langle e_{2 \beta}, A\right\rangle+2 h_{\alpha, i 2} h_{\beta, i 1}\left\langle e_{2 \alpha}, A\right\rangle\left\langle e_{1 \beta}, A\right\rangle \\
& -h_{\alpha, i 1} h_{\beta, i 1}\left\langle e_{1 \alpha}, A\right\rangle\left\langle e_{1 \beta}, A\right\rangle-h_{\alpha, i 2} h_{\beta, i 2}\left\langle e_{2 \alpha}, A\right\rangle\left\langle e_{2 \beta}, A\right\rangle \\
& -h_{\alpha, i 1} h_{\beta, i 2}\left\langle e_{1 \alpha}, A\right\rangle\left\langle e_{2 \beta}, A\right\rangle-h_{\alpha, i 2} h_{\beta, i 1}\left\langle e_{2 \alpha}, A\right\rangle\left\langle e_{1 \beta}, A\right\rangle \\
= & -|B|^{2} w^{2}-\sum_{i}\left(\sum_{\alpha}\left(h_{\alpha, i 1}\left\langle e_{1 \alpha}, A\right\rangle-h_{\alpha, i 2}\left\langle e_{2 \alpha}, A\right\rangle\right)\right)^{2} .
\end{aligned}
$$

Noting that $\Delta \log w=w^{-2}\left(w \Delta w-|\nabla w|^{2}\right)$, we arrive at 
Proposition 3.1. Let $M$ be a minimal submanifold of $\mathbb{R}^{n+m}$, with the rank of Gauss map at most 2 . If $w>0$ at a point, then locally

$$
\Delta \log w \leq-|B|^{2} .
$$

Remark 3.1. If $N$ is a 2-dimensional minimal submanifold in $S^{2+m}$, then the cone $C M$ over $M$ is a 3-dimensional minimal submanifold in $\mathbb{R}^{3+m}$. The rank of the Gauss map from CM is no more than 2, as will be shown in the next section. Hence Proposition [3.1 is a generalization of Proposition 2.2 in [6]. We note that Lemma 3.2 is also a generalization of Lemma 2.1 in [6].

Finally, we consider the general case. For any $x \in M$ satisfying $w(x)>0$, then $w\left(P, P_{0}\right)>0$ with $P=\gamma(x)$. Let $\theta_{1}, \cdots, \theta_{n}$ be the Jordan angles between $P$ and $P_{0}$ which are arranged as

$$
\frac{\pi}{2}>\theta_{1} \geq \cdots \geq \theta_{r}>\theta_{r+1}=\cdots=\theta_{n}=0
$$

with $0 \leq r \leq \min \{n, m\}$. As shown in Section 2, one can find an orthonormal basis $\left\{\varepsilon_{i}, \varepsilon_{n+\alpha}\right\}$ of $\mathbb{R}^{n+m}$, such that $\psi\left(P_{0}\right)=\varepsilon_{1} \wedge \cdots \wedge \varepsilon_{n}$; if we denote

$$
e_{i}:=\left\{\begin{array}{cc}
\cos \theta_{i} \varepsilon_{i}+\sin \theta_{i} \varepsilon_{n+i} & 1 \leq i \leq r \\
\varepsilon_{i} & r+1 \leq i \leq n
\end{array}\right.
$$

and

$$
\nu_{\alpha}:=\left\{\begin{array}{cc}
-\sin \theta_{\alpha} \varepsilon_{\alpha}+\cos \theta_{\alpha} \varepsilon_{n+\alpha} & 1 \leq \alpha \leq r \\
\varepsilon_{n+\alpha} & r+1 \leq \alpha \leq m
\end{array}\right.
$$

then $\left\{e_{i}, \nu_{\alpha}\right\}$ is also an orthonormal basis of $\mathbb{R}^{n+m}$ and $\psi(P)=e_{1} \wedge \cdots \wedge e_{n}$.

Now we use the notation

$$
v:=w^{-1}
$$

as in [26] and [17]; the function $v$ is well-defined in a neighborhood of $x$. By a direct calculation based on metric forms in terms of matrix coordinates (2.11), one can derive

$$
\Delta v=v|B|^{2}+v \sum_{i, j} 2 \lambda_{j}^{2} h_{j, i j}^{2}+v \sum_{i} \sum_{j \neq k} \lambda_{j} \lambda_{k}\left(h_{j, i j} h_{k, i k}+h_{k, i j} h_{j, i k}\right)
$$

as in [17]; where $\lambda_{j}=\tan \theta_{j}$ and $h_{\alpha, i j}=\left\langle B\left(e_{i}, e_{j}\right), \nu_{\alpha}\right\rangle$. Grouping the terms according to different types of the indices of the coefficients of the second fundamental form, one can proceed as in the proof of [17] Proposition 3.1 to get

$$
v^{-1} \Delta v \geq C_{1}|B|^{2} \quad \text { whenever } v \leq \beta<3 \text {. }
$$

Noting that $v=\exp (-\log w)$, one can immediately get

Proposition 3.2. If $M$ is a submanifold in $\mathbb{R}^{n+m}$ with parallel mean curvature, once $w \geq \frac{1}{3}+\delta$ with a positive number $\delta$, then

$$
\Delta \log w-|\nabla \log w|^{2} \leq-C_{1}|B|^{2} \quad \text { at } x
$$

with a positive constant $C_{1}$ depending only on $\delta$. 


\section{SubHARMONIC FUnCTIONS AND EXTRINSIC RIGIDITY PROBLEM}

We already defined the $S$-map $\mathscr{S}: \mathbf{G}_{n, m} \rightarrow \overline{\mathbb{D}}$ in $\$ 2$. For every $\left(x_{1}, x_{2}\right) \in$ $\overline{\mathbb{D}} \backslash\{(a, 0): a \leq 0\}$ that is obtained by deleting a radius from the disk, there exist unique $r \in(0,1]$ and $\theta \in(-\pi, \pi)$, such that

$$
\left(x_{1}, x_{2}\right)=(r \cos \theta, r \sin \theta) .
$$

Here $r$ and $\theta$ can be seen as smooth functions on $\mathscr{S}^{-1}(\overline{\mathbb{D}} \backslash\{(a, 0): a \leq 0\})$. For any $c \in[0,1)$, we put

$$
\mathbb{W}_{c}:=\mathscr{S}^{-1}\left(\overline{\mathbb{D}} \backslash\left(\overline{\mathbb{D}}_{c} \cup\{(a, 0): a \leq 0\}\right)\right),
$$

with $\overline{\mathbb{D}}_{c}=\left\{\left(x_{1}, x_{2}\right) \in \mathbb{R}^{2}: x_{1}^{2}+x_{2}^{2} \leq c^{2}\right\}$, then the function $r$ takes values between $c$ and 1 on $\mathbb{W}_{c}$.

Remark 4.1. If $m=1$, then $\mathbf{G}_{n, m}=S^{n}$ and $\mathbb{W}_{0}$ is the complement of a halfequator $\bar{S}_{+}^{n-1}$; in particular, if $n=2$, then $\mathbb{W}_{0}$ can be obtained by deleting half of a great circle between 2 antipodal points from $S^{2}$. It has been proved in $[16]$ that $\mathbb{W}_{0}$ is a maximal convex-supporting set in $S^{n}$.

Remark 4.2. Obviously, $P_{t}, P_{t+\pi} \in \mathbb{W}_{c}$ consist of an antipodal pair in $\mathbf{G}_{n, m}$ for each $t \in\left(-\frac{\pi}{2}, 0\right)$, which implies $w\left(P_{t}, S\right)=-w\left(P_{t+\pi}, S\right)$ for arbitrary $S \in \mathbf{G}_{n, m}$. Hence $\mathbb{W}_{c}$ cannot lie in an $(n \times m)$-matrix coordinate chart centered at any point of $\mathbf{G}_{n, m}$. Furthermore, they are conjugate to each other. Since the Grasmann manifolds are simply connected symmetric spaces of compact type, the cut point of $P_{t}$ along a geodesic coincides with the first conjugate point along the geodesic. Therefore, $\mathbb{W}_{c}$ contains a cut point of $P_{t}$ at least.

On $\mathbb{W}_{c}$ there are smooth functions $r$ and $\theta$. By the previous work [16] our concerned function $F$ was constructed from $r$ and $\theta$ and we need to estimate Hess $F$. Hopefully, the level set of $F=t$ coincides with level set of $w\left(\cdot, P_{t}\right)$. For estimating Hess $F$ in terms of Hess $w\left(\cdot, P_{t}\right)$ we need the following transition Lemma, which is crucial in our construction, but may also be useful in other contexts.

Lemma 4.1. Let $(N, h)$ be a Riemannian manifold, $F$ be a smooth function on $N$, and $\{H(\cdot, t): t \in \mathbb{R}\}$ be a smooth family of smooth functions on $N$. Assume that there are smooth real functions $t(s)$ and $l(s)$; for all $s \in \mathbb{R}$, the level set $F_{s}:=\{p \in$ $N: F(p)=s\}$ coincides with $\{p \in N: H(p, t(s))=l(s)\} ; \nabla F$ and $\nabla H(\cdot, t(s))$ are nonzero normal vector fields on $F_{s}$, pointing in the same direction. Then for arbitrary $\varepsilon>0$, there exists a smooth nonpositive function $\lambda$ on $N$, depending on $\varepsilon$, such that

$$
\text { Hess } F \geq \frac{|\nabla F|}{|\nabla H(\cdot, t(s))|} \operatorname{Hess} H(\cdot, t(s))-\varepsilon h+\lambda d F \otimes d F
$$

on $N$.

Proof. For any $s \in \mathbb{R}, F_{s}$ can be regarded as a hypersurface of $N$. Denote by $\nabla$ and $\nabla^{s}$ the Levi-Civita connection on $N$ and $F_{s}$, respectively. We put $\nu=\frac{\nabla F}{|\nabla F|}$, then $\nu$ 
is the unit normal vector field on $F_{s}$; and denote by $B$ the second fundamental form of $F_{s}$ in $N$.

For every $X \in T\left(F_{s}\right)$,

$$
\text { Hess } \begin{aligned}
F(X, X) & =\nabla_{X} \nabla_{X} F-\left(\nabla_{X} X\right) F \\
& =-\left(\nabla_{X}^{s} X\right) F-\langle B(X, X), \nu\rangle\langle\nu, \nabla F\rangle \\
& =-|\nabla F|\langle B(X, X), \nu\rangle .
\end{aligned}
$$

Similarly,

$$
\text { Hess } H(\cdot, t(s))(X, X)=-|\nabla H(\cdot, t(s))|\langle B(X, X), \nu\rangle \text {. }
$$

Hence

$$
\left(\operatorname{Hess} F-\frac{|\nabla F|}{|\nabla H(\cdot, t(s))|} \operatorname{Hess} H(\cdot, t(s))\right)(X, X)=0 \quad \text { for all } X \in T\left(F_{s}\right) \text {. }
$$

It implies the existence of a smooth 1 -form $\omega$ on $N$, such that

$$
\text { Hess } F-\frac{|\nabla F|}{|\nabla H(\cdot, t(s))|} \operatorname{Hess} H(\cdot, t(s))=\omega \otimes d F+d F \otimes \omega
$$

on $F_{s}$. Denote by $\omega^{*}$ the associated vector field of $\omega$ with respect to $h$, i.e.

$$
\left\langle Y, \omega^{*}\right\rangle=\omega(Y) \quad \text { for all } Y \in T N .
$$

Then

$$
\begin{aligned}
& (\omega \otimes d F+d F \otimes \omega)(Y, Y) \\
= & 2 \omega(Y) d F(Y)=2\left\langle\omega^{*}, Y\right\rangle d F(Y) \\
\geq & -2\left|\omega^{*}\right||Y| d F(Y) \geq-\varepsilon|Y|^{2}-\varepsilon^{-1}\left|\omega^{*}\right|^{2} d F(Y)^{2} .
\end{aligned}
$$

Let $\lambda=-\varepsilon^{-1}\left|\omega^{*}\right|^{2}$, then substituting (4.7) into (4.6) yields (4.2).

Given a compact subset $K$ of $\mathbb{W}_{c}$, there exists a positive number $\delta$, such that $r \geq c+2 \delta$ on $K$. Hence the following function

$$
F=\theta-\arccos \left(\frac{c+\delta}{r}\right)
$$

is well-defined on $K$. Once $F(S)=t$, one can deduce that $\theta-t=\arccos \left(\frac{c+\delta}{r}\right) \in$ $\left(0, \frac{\pi}{2}\right)$, and

$$
\begin{aligned}
c+\delta & =r(\cos (\theta-t))=r(\cos t \cos \theta+\sin t \sin \theta) \\
& =\left(\cos t x_{1}+\sin t x_{2}\right)(S)=w\left(S, P_{t}\right) .
\end{aligned}
$$

In other words, the level set $F_{t}$ of $F$ overlaps with the level set $\left\{S \in \mathbf{G}_{n, m}\right.$ : $\left.-\log w\left(S, P_{t}\right)=-\log (c+\delta)\right\}$.

To apply Lemma 4.1, we need gradient estimates for the functions $-\log w\left(\cdot, P_{t}\right)$ and $F$ along the common level set. 
Let $\mathbb{U}$ be the matrix coordinate chart centered at $P_{t}$. Recall that $w\left(\cdot, P_{t}\right)$ has an expression

$$
w=\left[\operatorname{tr}\left(I_{n}+Z Z^{T}\right)\right]^{-1}
$$

in terms of matrix coordinates (see [26]). It is easily seen that both $w$ and the metric on $\mathbb{U}$ are invariant under $S O(n) \times S O(m)$-actions. Hence for any $S \in \mathbb{U}$, without loss of generality one can assume

$$
Z(S)=\left(\lambda_{i} \delta_{i \alpha}\right)=\left(\tan \theta_{i} \delta_{i \alpha}\right)
$$

with $\left\{\theta_{i}\right\}$ being the Jordan angles between $S$ and $P_{t}$. As in [26], one can obtain

$$
d w\left(\cdot, P_{t}\right)=-\sum_{1 \leq i \leq p} \lambda_{i} w\left(\cdot, P_{t}\right) \omega_{i i} \quad \text { at } R
$$

with $p=\min \{n, m\}$ and $\left\{\omega_{i \alpha}\right\}$ is the dual basis of $\left\{\left(1+\lambda_{i}^{2}\right)^{\frac{1}{2}}\left(1+\lambda_{\alpha}^{2}\right)^{\frac{1}{2}} E_{i \alpha}\right\}$, which is an orthogonal basis of $T_{S} \mathbf{G}_{n, m}$. (Here $E_{i \alpha}$ is the matrix with 1 in the intersection of row $i$ and column $\alpha$ and 0 otherwise.) Therefore

$$
\left|\nabla \log w\left(\cdot, P_{t}\right)\right|^{2}=\sum_{1 \leq i \leq p} \lambda_{i}^{2} \geq p\left(w\left(\cdot, P_{t}\right)^{-\frac{2}{p}}-1\right)
$$

and the equality holds if and only if $\lambda_{1}^{2}=\cdots=\lambda_{p}^{2}=w\left(\cdot, P_{t}\right)^{-\frac{2}{p}}-1$. In particular, on $F_{t}$,

$$
\left|\nabla \log w\left(\cdot, P_{t}\right)\right|^{2} \geq p\left((c+\delta)^{-\frac{2}{p}}-1\right):=C_{2}(p, \delta)
$$

By $(2.15)$,

$$
\begin{aligned}
\nabla w\left(\cdot, P_{t}\right) & =\cos t \nabla x_{1}+\sin t \nabla x_{2}=\cos t \nabla(r \cos \theta)+\sin t \nabla(r \sin \theta) \\
& =\cos (t-\theta) \nabla r+r \sin (t-\theta) \nabla \theta
\end{aligned}
$$

and moreover by (4.9),

$$
\begin{aligned}
-\nabla \log w\left(\cdot, P_{t}\right) & =-w^{-1}\left(\cdot, P_{t}\right) \nabla w\left(\cdot, P_{t}\right)=-(c+\delta)^{-1} \nabla w\left(\cdot, P_{t}\right) \\
& =-r^{-1} \nabla r+\tan (\theta-t) \nabla \theta
\end{aligned}
$$

on $F_{t}$. On the other hand, from (4.8) we have

$$
\begin{aligned}
\nabla F & =\nabla \theta-\left(1-\left(\frac{c+\delta}{r}\right)^{2}\right)^{-\frac{1}{2}} \frac{c+\delta}{r^{2}} \nabla r \\
& =\nabla \theta-r^{-1} \cot (\theta-t) \nabla r .
\end{aligned}
$$

Comparing (4.13) with (4.14) gives

$$
-\nabla \log w\left(\cdot, P_{t}\right)=\tan (\theta-t) \nabla F \text {. }
$$

$c+2 \delta \leq r \leq 1$ gives $\theta-t=\arccos \left(\frac{c+\delta}{r}\right) \in\left[\arccos \left(\frac{c+\delta}{c+2 \delta}\right), \arccos (c+\delta)\right]$; hence $-\nabla \log w\left(\cdot, P_{t}\right)$ and $\nabla F$ point in the same direction, and

$$
\frac{\left|\nabla \log w\left(\cdot, P_{t}\right)\right|}{|\nabla F|}=\tan (\theta-t) \leq \frac{\sqrt{1-(c+\delta)^{2}}}{c+\delta}:=C_{3}(\delta) .
$$


Now we put $H(\cdot, t)=-\log w\left(\cdot, P_{t}\right)$, then by (4.12) and (4.16), one can apply Lemma 4.1 to get the following conclusion

Proposition 4.1. For a compact set $K \subset \mathbb{W}_{c}$, put $\delta:=\frac{1}{2} \inf _{K}(r-c)$ and $F:=$ $\theta-\arccos \left(\frac{c+\delta}{r}\right)$, then for arbitrary $\varepsilon>0$, there exists a smooth nonpositive function $\lambda$ on $K$, depending on $\varepsilon$, such that

$$
\text { Hess } F \geq-C_{3}^{-1} \text { Hess } \log w\left(\cdot, P_{t}\right)-\varepsilon g+\lambda d F \otimes d F
$$

on the level set $F_{t}$. Here $g$ is the canonical metric on $\mathbf{G}_{n, m}$ and $C_{3}$ is a positive constant depending only on $\delta$.

Remark 4.3. Put

$$
\begin{aligned}
\phi(t)\left(a_{i} e_{i}+b_{\alpha} e_{n+\alpha}\right)= & \left(a_{1} \cos t+b_{1} \sin t\right) e_{1}+\left(-a_{1} \sin t+b_{1} \cos t\right) e_{n+1} \\
& +\sum_{i \geq 2} a_{i} e_{i}+\sum_{\alpha \geq 2} b_{\alpha} e_{n+\alpha}
\end{aligned}
$$

then $t \in \mathbb{R} \mapsto \phi(t)$ is a 1-parameter subgroup of $S O(n+m)$, which induces a 1 parameter isometry group of $\mathbf{G}_{n, m}$. Since $w(\cdot, \cdot)$ is invariant under the action of $S O(n+m)$, it is easily-seen that $F_{t}$ is the orbit of $F_{0}$ under the action of $\phi(t)$. This observation can explain why the level sets of $F$ share similar properties in terms of exterior geometry.

Combining Propositions 3.1 3.2 and Proposition 4.1, we can find a strongly subharmonic function on a submanifold with parallel mean curvature under the assumptions on the Gauss image.

Proposition 4.2. Let $M$ be an oriented submanifold in $\mathbb{R}^{n+m}$ with parallel mean curvature, if its Gauss image is contained in a compact subset $K$ of $\mathbb{W}_{1 / 3}$, then there exists a positive bounded function $f$ on $M$, such that

$$
\Delta f \geq K_{0}|B|^{2}
$$

with a positive constant $K_{0}$ depending only on $K$. Moreover, if $M$ is minimal and the rank of the Gauss map is not larger than 2, then the above conclusion still holds when $\mathbb{W}_{1 / 3}$ is replaced by $\mathbb{W}_{0}$.

Proof. We only prove the general case, since the proof for the case with the additional assumption on the rank of the Gauss map is similar. gives

Using the definition of $F$ in (4.8) with $c=\frac{1}{3}$ and combining (4.17) and (4.16)

$$
\begin{aligned}
& \mu_{0}^{-1} \exp \left(\mu_{0} F\right) \operatorname{Hess} \exp \left(\mu_{0} F\right) \\
\geq & -C_{3}^{-1} \operatorname{Hess} \log w\left(\cdot, P_{t}\right)-\varepsilon g+\left(\lambda+\mu_{0}\right) d F \otimes d F \\
\geq & -C_{3}^{-1} \operatorname{Hess} \log w\left(\cdot, P_{t}\right)-\varepsilon g+C_{3}^{-2}\left|\lambda+\mu_{0}\right| d \log w\left(\cdot, P_{t}\right) \otimes d \log w\left(\cdot, P_{t}\right)
\end{aligned}
$$

where $\mu_{0}$ and $\varepsilon$ are positive constants to be chosen. Define

$$
f=\exp \left(\mu_{0} F\right) \circ \gamma
$$


with $\gamma$ the Gauss map into $K \subset \mathbb{W}_{1 / 3}$, then using the composition formula implies $(4.21)$

$$
\begin{aligned}
\mu_{0}^{-1} f^{-1} \Delta f= & \mu_{0}^{-1} \exp \left(\mu_{0} F\right)\left(\operatorname{Hess} \exp \left(\mu_{0} F\right)\left(\gamma_{*} e_{i}, \gamma_{*} e_{i}\right)+d \exp \left(\mu_{0} F\right)(\tau(\gamma))\right. \\
\geq & -C_{3}^{-1} \operatorname{Hess} \log w\left(\cdot, P_{t}\right)\left(\gamma_{*} e_{i}, \gamma_{*} e_{i}\right)-\varepsilon\left\langle\gamma_{*} e_{i}, \gamma_{*} e_{i}\right\rangle \\
& +C_{3}^{-2}\left|\lambda+\mu_{0}\right| \sum_{i}\left(\nabla_{e_{i}}\left|\log w\left(\cdot, P_{t}\right) \circ \gamma\right|\right)^{2} \\
= & -C_{3}^{-1} \Delta\left(\log w\left(\cdot, P_{t}\right) \circ \gamma\right)-\varepsilon|B|^{2}+C_{3}^{-2}\left|\lambda+\mu_{0}\right|\left|\nabla\left(\log w\left(\cdot, P_{t}\right) \circ \gamma\right)\right|^{2}
\end{aligned}
$$

on the level set $\left\{x \in M: f(x)=\exp \left(\mu_{0} t\right)\right\}$ with the tension field $\tau(\gamma)$ of the Gauss map $\gamma$. Here $\left\{e_{i}\right\}$ is an orthonormal basis of the tangent space at the considered point of $M$, and we have used the harmonicity of the Gauss map. Since $w\left(\cdot, P_{t}\right)$ equals $\frac{1}{3}+\delta$ everywhere on the level set $F_{t}$ (see (4.9)), one can apply Proposition 3.2 to obtain

$$
\Delta\left(\log w\left(\cdot, P_{t}\right) \circ \gamma\right)-\left|\nabla\left(\log w\left(\cdot, P_{t}\right) \circ \gamma\right)\right|^{2} \leq-C_{1}|B|^{2}
$$

with a positive constant $C_{1}$ depending only on $\delta$. Substituting this into (4.21) yields

$$
\mu_{0}^{-1} f^{-1} \Delta f \geq\left(C_{3}^{-1} C_{1}-\varepsilon\right)|B|^{2}+\left(C_{3}^{-2}\left|\lambda+\mu_{0}\right|-C_{3}^{-1}\right)\left|\nabla\left(\log w\left(\cdot, P_{t}\right) \circ \gamma\right)\right|^{2} .
$$

By letting $\varepsilon:=\frac{1}{2} C_{3}^{-1} C_{1}$ and $\mu_{0}:=\sup _{K}|\lambda|+C_{3}$ we arrive at (4.18) with $K_{0}=$ $\frac{1}{2} C_{3}^{-1} C_{1} \mu_{0} \exp \left(\mu_{0} \inf _{K} F\right)$.

We can immediately get the following Bernstein type theorem with the aid of the strongly subharmonic functions.

Theorem 4.1. If $M$ is a parabolic, oriented minimal surface in $\mathbb{R}^{2+m}$, and if its Gauss image is contained in a compact subset of $\mathbb{W}_{0}$, then $M$ has to be an affine linear subspace.

Proof. One can find a strongly subharmonic function $f$ on $M$ satisfying (4.18) by applying Proposition 4.2, since the mean curvature vector field vanishes and the rank of the Gauss map is $\leq 2$. Since a parabolic surface cannot admit any nonconstant bounded subharmonic function, $f$ has to be constant; hence $\Delta f \equiv 0$ and moreover $|B|^{2} \equiv 0$, which means $M$ is totally geodesic.

We now study the extrinsic rigidity problem, initiated by J. Simons [21], finding better conditions on the Gauss image for a compact minimal submanifold $M$ in $S^{n+m}$ to be an equator.

As pointed out and utilized by J. Simons [21], the properties of the (minimal) submanifolds $M$ in the sphere are closed related to those of the cone $C M$ generated by $M$, which is the image under the map of $M \times[0,+\infty) \rightarrow \mathbb{R}^{n+m+1}$ defined by 
$(x, t) \mapsto t x$. To avoid the singularity $t=0$, we also consider the truncated cone $C M_{\varepsilon}(\varepsilon>0)$ that is the image of $M \times(\varepsilon,+\infty)$ under the same map.

We choose a local orthonormal frame field $\left\{e_{i}, \nu_{\alpha}\right\}$ of $S^{n}$ along $M$, then by parallel translating along rays issuing from the origin we obtain local vector fields $\left\{E_{i}\right\}$ and $\left\{\mathcal{N}_{\alpha}\right\}$; obviously $E_{i}=\frac{1}{t} e_{i}$ and $\mathcal{N}_{\alpha}=\frac{1}{t} \nu_{\alpha}$. Let $\tau$ be the unit tangent vector along the rays, i.e. $\tau=\frac{\partial}{\partial t}$, then $\left\{E_{i}, \mathcal{N}_{\alpha}, \tau\right\}$ is a local orthonormal frame field in $\mathbb{R}^{n+m+1}$ and $\left\{E_{i}, \tau\right\}$ is a frame field in $C M_{\varepsilon}$.

Let $B$ and $B^{c}$ denote the second fundamental form of $M \subset S^{n+m}$ and $C M_{\varepsilon} \subset$ $\mathbb{R}^{n+m+1}$, respectively. Then a straightforward calculation shows (see [23] p.18)

$$
\left\langle B^{c}\left(E_{i}, E_{j}\right), \mathcal{N}_{\alpha}\right\rangle=\frac{1}{t}\left\langle B\left(e_{i}, e_{j}\right), \nu_{\alpha}\right\rangle
$$

and

$$
B^{c}\left(E_{i}, \tau\right)=B^{c}(\tau, \tau)=0
$$

Hence $C M_{\varepsilon}$ is a minimal submanifold in $\mathbb{R}^{n+m+1}$ if and only if $M$ is a minimal submanifold in $S^{n+m}$.

The normal Gauss map $\gamma^{N}: M \rightarrow \mathbf{G}_{m, n+1}$ is defined by

$$
\gamma^{N}(x)=N_{x} M
$$

via parallel translation in $\mathbb{R}^{n+m+1}$. Let $\eta$ be the natural isometry between $\mathbf{G}_{n+1, m}$ and $\mathbf{G}_{m, n+1}$ which maps each $(n+1)$-dimensional oriented linear subspace to its orthogonal complementary $m$-space. It is easily seen that the Gauss map $\gamma: C M_{\varepsilon} \rightarrow$ $\mathbf{G}_{n+1, m}$ is a cone-like map; more precisely, for every $t \in(\varepsilon,+\infty)$ and $x \in M$,

$$
(\eta \circ \gamma)(t x)=\gamma^{N}(x)
$$

Thus $\gamma_{*} \tau=0$ and the rank of $\gamma$ at $t x$ equals the rank of $\gamma^{N}$ at $x$ for all $x \in M$ and any $t \in(\varepsilon,+\infty)$.

One can assume $\nabla e_{i}=0$ at the considered point without loss of generality, then by computing (see [23] p.18),

$$
\nabla_{E_{i}}^{c} E_{j}=-\frac{1}{t} \delta_{i j} \tau
$$

with $\nabla^{c}$ the Levi-Civita connection on $C M_{\varepsilon}$. Let $f$ be a cone-like real function on $C M_{\varepsilon}$, then

$$
\Delta^{c} f=\nabla_{E_{i}}^{c} \nabla_{E_{i}}^{c} f-\left(\nabla_{E_{i}}^{c} E_{i}\right) f=\frac{1}{t^{2}} \nabla_{e_{i}} \nabla_{e_{i}} f_{1}+\frac{1}{t} \nabla_{\tau}^{c} f=\frac{1}{t^{2}} \Delta f_{1} .
$$

Here $\Delta^{c}$ is the Laplace-Beltrami operator on $C M_{\varepsilon}$ and $f_{1}$ is a function on $M$ satisfying $f(t x)=f_{1}(x)$ for every $x \in M$ and $t \in(\varepsilon,+\infty)$.

Based on the strongly subharmonic functions constructed in Proposition 4.2, we can derive the following extrinsic rigidity result. 
Theorem 4.2. Let $M^{n}$ be a compact, oriented minimal submanifold in $S^{n+m}, P, Q \in$ $\mathbf{G}_{m, n+1}$ that are $S$-orthogonal to each other. Assume

$$
w(N, P)^{2}+w(N, Q)^{2}>\left\{\begin{array}{cc}
0 & \text { if } \operatorname{rank}\left(\gamma^{N}\right) \leq 2 \\
\frac{1}{9} & \text { otherwise }
\end{array}\right.
$$

holds for all normal $m$-vectors $N$ of $M$, and there exists no point $x \in M$, such that $w(N, Q)=0$ and $w(N, P)<0$ at $x$. Then $M$ has to be an equator.

Proof. As above, we only give the proof for the general case without the assumptions on the rank of the Gauss map.

By the definition of $\eta$, we have

$$
\psi(\eta(S))=*(\psi(S))
$$

with $\psi$ denoting the Plücker embedding and * being the Hodge star operator. * keeps the inner product invariant, i.e.

$$
\langle * A, * B\rangle=\langle A, B\rangle \quad \text { for } A, B \in \Lambda^{m}\left(\mathbb{R}^{n+m+1}\right) .
$$

Hence

$$
w\left(\eta\left(S_{1}\right), \eta\left(S_{2}\right)\right)=w\left(S_{1}, S_{2}\right) \quad \text { for } S_{1}, S_{2} \in \mathbf{G}_{m, n+1} .
$$

If $S_{1}, S_{2}$ are S-orthogonal to each other, then $w\left(S_{1}, S_{2}\right)=0$ and $\operatorname{dim}\left(S_{1} \cap S_{2}\right)=m-1$, which implies $w\left(\eta\left(S_{1}\right), \eta\left(S_{2}\right)\right)=0$ and $\operatorname{dim}\left(\eta\left(S_{1}\right)+\eta\left(S_{2}\right)\right)=n+m+1-\operatorname{dim}\left(S_{1} \cap\right.$ $\left.S_{2}\right)=n+2$, hence $\eta\left(S_{1}\right)$ and $\eta\left(S_{2}\right)$ are S-orthogonal to each other (see Proposition 2.1).

Denote

$$
\mathbb{W}_{\frac{1}{3}}=\left\{S \in \mathbf{G}_{n+1, m}:(w(S, \eta(P)), w(S, \eta(Q))) \in \mathbb{D} \backslash\left(\overline{\mathbb{D}}_{\frac{1}{3}} \cup\{(a, 0): a \leq 0\}\right)\right\},
$$

then the assumption on the normal Gauss map $\gamma^{N}$ implies that the image under the Gauss map of $C M_{\varepsilon}$ is contained in a compact subset of $\mathbb{W}_{1 / 3}$. This enables us to find a strongly subharmonic function $f$ satisfying

$$
\Delta^{c} f \geq K_{0}\left|B^{c}\right|^{2} \text {. }
$$

Noting that $f$ is the composition of the Gauss map $\gamma$ and a function on $K$, it should be a cone-like function. Denote $f_{1}=\left.f\right|_{M}$, then combining (4.28), (4.26) and (4.23) gives

$$
\Delta f_{1}=t^{2} \Delta^{c} f \geq K_{0} t^{2}\left|B^{c}\right|^{2}=K_{0}|B|^{2} .
$$

Integrating both sides along $M$ implies

$$
0=\int_{M} \Delta f_{1} * 1 \geq K_{0} \int_{M}|B|^{2} * 1
$$

Thus $|B|^{2} \equiv 0$ and $M$ has to be totally geodesic.

Remark 4.4. Obviously $w(N, P)>0$ implies $w(N, P)^{2}+w(N, Q)^{2}>0$. Hence for the case of dimension 2, our result is an improvement of extrinsic rigidity theorems of Barbosa [2] and Fischer-Colbrie [6] . 


\section{Curvature estimates}

In the previous section, we have found a strongly subharmonic function $f$ on a submanifold $M^{n} \subset \mathbb{R}^{n+m}$ with parallel mean curvature under the assumption on the Gauss image. To make Stokes' theorem applicable, we take a $H^{1,2}$-function $\eta$ with compact support; multiplying $\eta$ with both sides of (4.18) and then integrating it along $M$ gives

$$
\begin{aligned}
K_{0} \int_{M}|B|^{2} \eta * 1 & \leq \int_{M} \eta \Delta f * 1=\int_{M}(\operatorname{div}(\eta \nabla f)-\langle\nabla \eta, \nabla f\rangle) * 1 \\
& =-\int_{M}\langle\nabla \eta, \nabla f\rangle * 1 .
\end{aligned}
$$

In order to obtain a-priori estimates for $|B|^{2}$, we have to choose a 'good' test function $\eta$.

We shall use the Green test function technique employed in [11] and [12]. Unfortunately, this method cannot be applied for arbitrary noncompact Riemannian manifolds. We have to impose a so-called DVP-condition as in [16].

Definition 5.1. Let $(M, g)$ be a Riemannian manifold (not necessarily complete) satisfying:

(D) There is a distance function $d$ on $M$, such that the metric topology induced by $d$ is equivalent to the Riemannian topology of $M$, and $d(x, y) \leq r(x, y)$ for any $x, y \in M$, where $r$ is the distance function induced by the metric tensor of $M$;

$(V)$ Let $B_{R}\left(x_{0}\right)=\left\{x \in M: d\left(x, x_{0}\right)<R\right\}, V\left(x_{0}, R\right)=\operatorname{Vol}\left(B_{R}\left(x_{0}\right)\right)$, then there exists a positive constant $K_{1}$ not depending on $R$ and $x_{0}$, such that

$$
V\left(x_{0}, 2 R\right) \leq K_{1} V\left(x_{0}, R\right) \quad \text { whenever } B_{2 R}\left(x_{0}\right) \subset \subset M
$$

(P) For any $x_{0} \in M$ and $R>0$ satisfying $B_{R}\left(x_{0}\right) \subset \subset M$, the following NeumannPoincaré inequality

$$
\int_{B_{R}\left(x_{0}\right)}\left|v-\bar{v}_{R}\right|^{2} * 1 \leq K_{2} R^{2} \int_{B_{R}\left(x_{0}\right)}|\nabla v|^{2} * 1
$$

holds with $\bar{v}_{R}$ denoting the average value of $v$ in $B_{R}\left(x_{0}\right)$ and $K_{2}$ being a positive constant not depending on $x_{0}$ and $R$.

Then we say that $(M, g)$ satisfies a DVP-condition.

Remark 5.1. If $M=\mathbb{D}^{n}\left(r_{0}\right) \subset \mathbb{R}^{n}$ with metric $g=g_{i j} d x^{i} d x^{j}$, and the eigenvalues of $\left(g_{i j}\right)$ are uniformly bounded from below by a positive constant $\lambda$ and from above by a positive constant $\mu$, then by denoting $d(x, y)=\lambda|x-y|$ with $|\cdot|$ the standard Euclidean norm, we can show $(M, g)$ satisfies the DVP-condition with $K_{1}=\left(\frac{4 \mu}{\lambda}\right)^{n}$ and $K_{2}=4 \pi^{-2}\left(\frac{\mu}{\lambda}\right)^{n+2}$ (see [16]). Thus every Riemannian manifold has a coordiate chart centered at any point that satisfies the DVP-condition. If $r_{0}=+\infty$, we call $M$ a simple manifold. Other complete Riemannian manifolds satisfying a DVPcondition include $(M, g)$ with nonnegative Ricci curvature (see [4] for the proof of the Neumann-Poincaré inequality) and area-minimizing embedded hypersurfaces in $\mathbb{R}^{n+1}$. (Here $d$ is taken to be the extrinsic distance function, see [3] for the proof of the Neumann-Poincaré inequality.) 
Based on the DVP-condition, one can apply Moser's iteration [19] to derive the Harnack inequality for superharmonic functions on $M$ (see [16] Lemma 4.1), which implies the following estimates:

Lemma 5.1. ([16], Corollary 4.1) Let $M$ be a Riemannian manifold satisfying a DVP-condition, then there exists a constant $C_{4} \in(0,1)$, only depending on $K_{1}$ and $K_{2}$, such that

$$
v_{+, \frac{R}{2}} \leq\left(1-C_{4}\right) v_{+, R}+C_{4} \bar{v}_{\frac{R}{2}}
$$

for any subharmonic function $v$ on $B_{R}\left(x_{0}\right)\left(B_{2 R}\left(x_{0}\right) \subset \subset M\right)$. Here $v_{+, R}$ denotes the supremum of $v$ on $B_{R}\left(x_{0}\right)$.

Let $\Omega$ be an open domain of $M$ and $\rho>0$. Using the Lax-Milgram Theorem, it is easy to prove that there is a unique $H_{0}^{1,2}(\Omega)$-function $G^{\rho}(\cdot, x)$, such that

$$
\int_{\Omega}\left\langle\nabla G^{\rho}(\cdot, x), \nabla \phi\right\rangle * 1=f_{B_{\rho}(x)} \phi \quad \text { for any } \phi \in H_{0}^{1,2}(\Omega)
$$

whenever $B_{\rho}(x) \subset \Omega$. Here

$$
f_{B_{\rho}(x)} \phi=\frac{\int_{B_{\rho}(x)} \phi}{V(x, \rho)} .
$$

$G^{\rho}$ is called the mollified Green function. As in [9] and [16], we can obtain a-priori estimates for mollified Green functions:

Lemma 5.2. (16], Lemma 4.3) Let $(M, g)$ be a Riemannian manifold (dimension $\geq 3)$ satisfying a DVP-condition. Let $x_{0} \in M$ and $R>0$ satisfying $B_{2 R}\left(x_{0}\right) \subset \subset M$. Denote

$$
\omega^{R}=\frac{V\left(x_{0}, \frac{R}{2}\right)}{R^{2}} G^{\frac{R}{2}}\left(\cdot, x_{0}\right)
$$

with $G^{\frac{R}{2}}$ being the mollified Green function on $B_{R}\left(x_{0}\right)$, then

$$
\omega^{R} \leq c_{1} \quad \text { on } B_{R}\left(x_{0}\right)
$$

and

$$
\omega^{R} \geq c_{2} \quad \text { on } B_{\frac{R}{2}}\left(x_{0}\right)
$$

with $c_{1}$ and $c_{2}$ denoting positive constants depending only on $K_{1}$ and $K_{2}$, not depending on $x_{0}$ and $R$.

Choosing $\eta=\left(\omega^{R}\right)^{2}$ as a test function in (5.1), we can derive the following curvature estimates with the aid of Lemmas [5.1 and 5.2. See [16] and [17] for details.

Lemma 5.3. (Curvature estimates) Let $M^{n}$ be a submanifold in $\mathbb{R}^{n+m}(n \geq 3)$ with parallel mean curvature. Assume there is a distance function $d$ on $M$, the metric ball $B_{4 R_{0}}\left(x_{0}\right)$ defined by $d$ satisfies the DVP-condition for some $x_{0} \in M$ and $R_{0} \in(0,+\infty]$, and the Gauss image of $B_{4 R_{0}}\left(x_{0}\right)$ is contained in a compact set 
$K \subset \mathbb{W}_{1 / 3}$. Then there exists a positive constant $C_{5}$, depending only on $K, K_{1}, K_{2}$, such that for arbitrary $R<R_{0}$,

$$
\frac{R^{2}}{V\left(x_{0}, R\right)} \int_{B_{R}\left(x_{0}\right)}|B|^{2} * 1 \leq C_{5}\left(f_{+, R}-f_{+, \frac{R}{2}}\right)
$$

with $f$ being the strongly subharmonic function constructed in Proposition 4.2. Moreover, there exists a positive constant $C_{6}$ depending only on $K, K_{1}, K_{2}$, such that for arbitrary $\varepsilon>0$, we can find $R \in\left[\exp \left(-C_{6} \varepsilon^{-1}\right) R_{0}, R_{0}\right]$, such that

$$
\frac{R^{2}}{V\left(x_{0}, R\right)} \int_{B_{R}\left(x_{0}\right)}|B|^{2} * 1 \leq \varepsilon .
$$

If $M$ is a minimal submanifold with $\operatorname{rank}(\gamma) \leq 2$, then the condition on the Gauss image can be relaxed to $\gamma\left(B_{4 R_{0}}\left(x_{0}\right)\right) \subset K \subset \mathbb{W}_{0}$.

\section{Gauss image Shrinking Lemmas and Bernstein type theOREMS}

Let $u$ be a harmonic map from $M$ and $H$ be a smooth function on the target manifold, then $H \circ u$ defines a smooth function on $M$. Take a cut-off function $\eta$ supported in the interior of $B_{R}\left(x_{0}\right), 0 \leq \eta \leq 1, \eta \equiv 1$ on $B_{\frac{R}{2}}\left(x_{0}\right)$ and $|\nabla \eta| \leq c_{0} R^{-1}$; and denote by $G^{\rho}$ the mollified Green function in $B_{R}\left(x_{0}\right)$. Then by Stokes' theorem

$$
\int_{B_{R}\left(x_{0}\right)} \operatorname{div}\left(\eta G^{\rho}(\cdot, x) \nabla(H \circ u)\right) * 1=0 \quad \text { whenever } B_{\rho}(x) \subset B_{R}\left(x_{0}\right) .
$$

After careful calculation as in [16] [17, we can arrive at a pointwise estimate for $H \circ u$ :

Proposition 6.1. Let $M$ be as in Lemma 5.3. Let $u$ be a harmonic map of $B_{4 R_{0}}\left(x_{0}\right) \subset M$ into a convex domain $V \subset \mathbb{R}^{l}$ with metric $h_{i j} d x^{i} d x^{j}$, such that

$$
K_{3}|\xi|^{2} \leq \xi^{i} h_{i j} \xi^{j} \leq K_{4}|\xi|^{2}
$$

everywhere on $V$. Then for any smooth function $H$ on $V$,

$$
\begin{aligned}
H \circ u(x) \leq & H\left(\bar{u}_{R}\right)+C_{7} \sup _{V}|\nabla H|\left(\frac{R^{2}}{V\left(x_{0}, R\right)} \int_{B_{R}\left(x_{0}\right)}|d u|^{2} * 1\right)^{\frac{1}{2}} \\
& -\liminf _{\rho \rightarrow 0^{+}} \int_{B_{R}\left(x_{0}\right)} G^{\rho}(\cdot, x) \eta \Delta(H \circ u) * 1
\end{aligned}
$$

for all $x \in B_{\frac{R}{4}}\left(x_{0}\right)$ with $R<R_{0}$. Here $\bar{u}_{R} \in \bar{V}$ (the closure of $V$ ) denotes the average value of $u$ in $B_{R}\left(x_{0}\right)$ and $C_{7}$ is a positive constant depending only on $K, K_{1}, K_{2}, K_{3}, K_{4}$, but independent of $H, x_{0}$ and $R$.

If we take $u$ to be the harmonic Gauss map, then $|d u|^{2}=|d \gamma|^{2}=|B|^{2}$, and Lemma 5.3 implies that the second term on the right hand side of (6.2) becomes arbitrarily small when $R$ is sufficiently small. Hence, for proving the Gauss image shrinking property as in [16][17] it remains to show: 
- $\mathbb{W}_{0}$ is diffeomorphic to a convex domain in Euclidean space;

- For any given $S \in K \subset \mathbb{W}_{1 / 3}$, one can find a smooth function $H$, such that $H \circ \gamma$ is a subharmonic function on $B_{R}\left(x_{0}\right)$, and the sublevel set $(H \leq H(S))$ is contained in a matrix coordinate chart of some point in $\mathbf{G}_{n, m}$.

In the sequel, $x_{1}, x_{2}, r, \theta, e_{i}, e_{n+\alpha}$ and $P_{t}$ will be as in Section 4 .

Lemma 6.1. There is a diffeomorphism $\phi=\left(\phi_{1}, \phi_{2}\right): \mathbb{W}_{0} \rightarrow(-\pi, \pi) \times \mathbb{R}^{n m-1}$ defined by

$$
S \mapsto\left(\phi_{1}(S), \phi_{2}(S)\right)
$$

such that

$$
\begin{aligned}
& \phi_{1}=\theta \\
& \left|\phi_{2}(S)\right|=\frac{1}{r}-1 .
\end{aligned}
$$

Proof. As shown in Section $4, \theta$ is a smooth $(-\pi, \pi)$-valued function on $\mathbb{W}_{0}$, and we shall investigate the level sets of $\theta$.

For any $t \in(-\pi, \pi)$, when $\theta(S)=t$ we have $w\left(S, P_{t+\frac{\pi}{2}}\right)=0$ and $w\left(S, P_{t}\right)>0$ by (2.15). Now we write

$$
y_{1}=w\left(\cdot, P_{t}\right), \quad y_{2}=w\left(\cdot, P_{t+\frac{\pi}{2}}\right)
$$

then

$$
y_{1}=\cos t x_{1}+\sin t x_{2} \quad y_{2}=-\sin t x_{1}+\cos t x_{2} .
$$

Denote $\mathbb{U}_{t}=\left\{S \in \mathbf{G}_{n, m}: w\left(S, P_{t}\right)>0\right\}$. Let $\chi_{t}: \mathbb{U}_{t} \rightarrow M_{n \times m}$ be the diffeomorphism mapping $S \in \mathbb{U}_{t}$ to its $(n \times m)$-matrix coordinate, then the Plücker coordinate of $S$ is

$$
\psi(S)=\left(f_{1}+Z_{1 \alpha} f_{n+\alpha}\right) \wedge \cdots \wedge\left(f_{n}+Z_{n \alpha} f_{n+\alpha}\right) \quad \text { with } Z=\chi_{t}(S) .
$$

Here $f_{1}=\cos t e_{1}+\sin t e_{n+1}, f_{n+1}=-\sin t e_{1}+\cos t e_{n+1}, f_{i}=e_{i}$ for all $2 \leq i \leq n$ and $f_{n+\alpha}=e_{n+\alpha}$ for every $2 \leq \alpha \leq m$. Noting that $\psi\left(P_{t}\right)=f_{1} \wedge \cdots \wedge f_{n}$ and $\psi\left(P_{t+\frac{\pi}{2}}\right)=f_{n+1} \wedge f_{2} \wedge \cdots \wedge f_{n}$. A direct calculation shows that for $S \in U_{t}$

$$
y_{1}(S)=w\left(S, P_{t}\right)=\frac{\left\langle\psi(S), \psi\left(P_{t}\right)\right\rangle}{\langle\psi(S), \psi(S)\rangle^{\frac{1}{2}}\left\langle\psi\left(P_{t}\right), \psi\left(P_{t}\right)\right\rangle^{\frac{1}{2}}}=\left(I_{n}+Z Z^{T}\right)^{-\frac{1}{2}},
$$

and

$$
y_{2}(S)=w\left(S, P_{t+\frac{\pi}{2}}\right)=\frac{\left\langle\psi(S), \psi\left(P_{t+\frac{\pi}{2}}\right)\right\rangle}{\langle\psi(S), \psi(S)\rangle^{\frac{1}{2}}\left\langle\psi\left(P_{t+\frac{\pi}{2}}\right), \psi\left(P_{t+\frac{\pi}{2}}\right)\right\rangle^{\frac{1}{2}}}=Z_{11}\left(I_{n}+Z Z^{T}\right)^{-\frac{1}{2}} .
$$

Hence, $y_{2}(R)=0$ if and only if $Z_{11}=0$, i.e.

$$
\left\{S \in \mathbb{W}_{0}: \theta(S)=t\right\}=\chi_{t}^{-1}\left\{Z \in M_{n \times m}: Z_{11}=0\right\}
$$

A straightforward calculation gives

$$
\begin{aligned}
& \nabla y_{1}=\cos (t-\theta) \nabla r+r \sin (t-\theta) \nabla \theta, \\
& \nabla y_{2}=-\sin (t-\theta) \nabla r+r \cos (t-\theta) \nabla \theta .
\end{aligned}
$$


Hence, along the level set $(\theta=t)$

$$
r=y_{1}, \quad \nabla y_{1}=\nabla r, \quad \nabla y_{2}=r \nabla \theta .
$$

Differentiating both sides of (6.7) and noting (6.8), we have

$$
\nabla \theta=r^{-1} \nabla y_{2}=r^{-1} \nabla\left(Z_{11} y_{1}\right)=r^{-1}\left(y_{1} \nabla Z_{11}+Z_{11} \nabla y_{1}\right)=\nabla Z_{11},
$$

whenever $\theta(S)=t$. In $U_{t}$ let $E_{i \alpha}$ be the matrix with 1 in the intersection of row $i$ and column $\alpha$ and 0 otherwise. Denote $g_{i \alpha, j \beta}=\left\langle E_{i \alpha}, E_{j \beta}\right\rangle$ and let $\left(g^{i \alpha, j \beta}\right)$ be the inverse matrix of $\left(g_{i \alpha, j \beta}\right)$. A standard computation shows $\left|\nabla Z_{11}\right|^{2}=g^{11,11}$. It has been shown in [26] that the eigenvalues of $\left(g^{i \alpha, j \beta}\right)$ are

$$
\left\{\left(1+\lambda_{i}^{2}\right)\left(1+\lambda_{\alpha}^{2}\right): 1 \leq i \leq n, 1 \leq \alpha \leq m\right\}
$$

with $\lambda_{i}=\tan \theta_{i}$ and $\left\{\theta_{i}\right\}$ being the Jordan angles between $S$ and $P_{t}$. Hence

$$
1 \leq g^{11,11} \leq \max _{(i, \alpha)}\left(\left(1+\lambda_{i}^{2}\right)\left(1+\lambda_{\alpha}^{2}\right)\right) \leq w^{-4}\left(\cdot, P_{t}\right)=r^{-4}
$$

i.e.

$$
1 \leq|\nabla \theta|^{2} \leq r^{-4}
$$

From (6.8) and (6.10), we see that $\theta$ is a non-degenerate function on $\mathbb{W}_{0}$, and each level set of $\theta$ is diffeomorphic to $\mathbb{R}^{n m-1}$. T: $M_{n \times m} \rightarrow M_{n \times m}$ defined by

$$
Z \mapsto\left(\operatorname{det}\left(I_{n}+Z Z^{T}\right)^{\frac{1}{2}}-1\right) \frac{Z}{\left(\operatorname{tr}\left(Z Z^{T}\right)\right)^{\frac{1}{2}}}
$$

obviously is a diffeomorphism. Note that here $\left(\operatorname{tr}\left(Z Z^{T}\right)\right)^{\frac{1}{2}}=\left(\sum_{i, \alpha} Z_{i \alpha}^{2}\right)^{\frac{1}{2}}$ equals the Euclidean norm of $Z$ when $Z$ is treated as a vector in $\mathbb{R}^{n m}$. Thus, $T \circ \chi_{t}$ is a diffeomorphism between the level set $(\theta=t)$ and $\mathbb{R}^{n m-1}$, moreover

$$
\left|T \circ \chi_{t}\right|=w^{-1}\left(\cdot, P_{t}\right)-1=r^{-1}-1 .
$$

Therefore, $\phi_{1}=\theta$ and $\phi_{2}=T \circ \chi_{t}$ are the required mappings.

Proposition 6.2. For any $c \in\left(\frac{1}{3}, 1\right)$ and any compact subset $\Theta$ of $(-\pi, \pi)$, there exists a smooth family of nonnegative, smooth functions $H(\cdot, t)(t \in \Theta)$ on

$$
\overline{\mathbb{W}}_{c, \Theta}:=\left\{S \in \mathbb{W}_{0}: r(S) \geq c, \theta(S) \in \Theta\right\},
$$

such that

(i) $H(S, t)=0$ if and only if $S=P_{t}$;

(ii) $H(S, t) \leq 1$ (or $H(S, t)<1)$ if and only if $w\left(S, P_{t}\right) \geq \frac{3}{4} c+\frac{1}{12} \quad$ or $w\left(S, P_{t}\right)>$ $\left.\frac{3}{4} c+\frac{1}{12}\right)$

(iii) For any submanifold $M$ in $\mathbb{R}^{n+m}$ with parallel mean curvature, if the Gauss image of $M$ is contained in $\overline{\mathbb{W}}_{c, \Theta}$, then $H(\cdot, t) \circ \gamma$ is a subharmonic function on $M$ for all $t \in \Theta$. 
Proposition 6.3. For any $c \in(0,1)$ and any compact subset $\Theta$ of $(-\pi, \pi)$, there exists a smooth family of nonnegative, smooth functions $H(\cdot, t)(t \in \Theta)$ on $\overline{\mathbb{W}}_{c, \Theta}$, such that

(i) $H(S, t)=0$ if and only if $S=P_{t}$;

(ii) $H(S, t) \leq 1$ (or $H(S, t)<1)$ if and only if $w\left(S, P_{t}\right) \geq \frac{3}{4} c\left(\right.$ or $\left.w\left(S, P_{t}\right)>\frac{3}{4} c\right)$;

(iii) If $M$ is a minimal submanifold in $\mathbb{R}^{n+m}$ with $\operatorname{rank}(\gamma) \leq 2$, then $H(\cdot, t) \circ \gamma$ is a subharmonic function on $M$ for all $t \in \Theta$ if the Gauss image of $M$ is contained in $\overline{\mathbb{W}}_{c, \Theta}$.

Proof. We only prove Proposition 6.2, since the proof of Proposition 6.3 is similar.

Let $\varphi$ be a smooth function on $[0,+\infty)$ satisfying

$$
\begin{cases}\varphi(u)=0, & u \in\left[0, \frac{11}{12}-\frac{3}{4} c\right] \\ \varphi(u)=u-\frac{5}{6}+\frac{1}{2} c, & u \in\left[\frac{3}{4}-\frac{1}{4} c,+\infty\right) \\ 0 \leq \varphi^{\prime} \leq 1 & \end{cases}
$$

Then one can define $\Psi$ on $\overline{\mathbb{W}}_{c, \Theta} \times(-\pi, \pi) \times[0,+\infty)$ by

$$
(S, t, u) \mapsto \begin{cases}r \cos (\theta-t-\varphi(u))+u-\varphi(u)-1 & \text { if } \theta(S) \geq t \\ r \cos (\theta-t+\varphi(u))+u-\varphi(u)-1 & \text { if } \theta(S)<t\end{cases}
$$

Now we fix $t$ and denote $\Psi_{t}(S, u)=\Psi(S, t, u)$. For arbitrary $S \in \overline{\mathbb{W}}_{c, \Theta}$, we put

$$
I_{S}:=\{u \in[0,+\infty): \max \{0,|\theta-t|-\pi\} \leq \varphi(u) \leq|\theta-t|\}
$$

then obviously $I_{S}$ is a closed interval, $I_{S}:=\left[m_{S}, M_{S}\right]$. If $\theta(S) \geq t$, then

$$
\partial_{2} \Psi_{t}=1-(1+r \sin (\theta-t-\varphi(u))) \varphi^{\prime}(u) \geq 0
$$

and the equality holds if and only if $\varphi(u)=|\theta-t|$ or $|\theta-t|-\pi$ and $\varphi^{\prime}(u)=1$; which implies $\varphi(u)=m_{S}$ or $M_{S}$. Thus

$$
\partial_{2} \Psi_{t}(S, \cdot)>0 \quad \text { on }\left(m_{S}, M_{S}\right) .
$$

Similarly (6.12) holds when $\theta(S)<t$.

When $|\theta(S)-t| \leq \pi$, we have $m_{S}=0$ and hence

$$
\Psi_{t}\left(S, m_{S}\right)=r \cos (\theta-t)-1 \leq 0
$$

Otherwise $|\theta(S)-t|>\pi$ and $m_{S}$ satisfies $\varphi\left(m_{S}\right)=|\theta-t|-\pi$, thus

$$
\begin{aligned}
\Psi_{t}\left(S, m_{S}\right) & =-r+m_{S}-\varphi\left(m_{S}\right)-1 \leq-r-1+\lim _{u \rightarrow+\infty}(u-\varphi(u)) \\
& =-r-1+\frac{5}{6}-\frac{1}{2} c \leq-\frac{3}{2} c-\frac{1}{6}<0 .
\end{aligned}
$$

Here the first inequality follows from $(u-\varphi(u))^{\prime}=1-\varphi^{\prime}(u) \geq 0$. 
Obviously $\varphi\left(M_{S}\right)=|\theta-t|$. By the definition of $\varphi, \varphi$ cannot be identically zero on a neighborhood of $M_{S}$. Thus $M_{S} \geq \frac{11}{12}-\frac{3}{4} c$, and

$$
\begin{aligned}
\Psi_{t}\left(S, M_{S}\right) & =r+M_{S}-\varphi\left(M_{S}\right)-1 \geq r-1+\frac{11}{12}-\frac{3}{4} c \\
& \geq \frac{1}{4} c-\frac{1}{12}>0 .
\end{aligned}
$$

By (6.12)-(6.15), for each $S \in \overline{\mathbb{W}}_{c, \Theta}$, there exists a unique $\tilde{H}=\tilde{H}(S, t) \in$ $\left[m_{S}, M_{S}\right)$, such that

$$
\Psi(S, t, \tilde{H}(S, t))=\Psi_{t}(S, \tilde{H}(S, t))=0
$$

Denote

$$
\Omega:=\left\{(S, t) \in \overline{\mathbb{W}}_{c, \Theta} \times(-\pi, \pi): \theta(S) \neq t\right\},
$$

then $\Psi$ is obviously smooth on $\Omega \times[0,+\infty)$. Hence the implicit theorem implies $\tilde{H}$ is smooth on $\Omega$. To show smoothness of $\tilde{H}$, it remains to prove that $\tilde{H}$ is smooth on a neighborhood of $\Omega^{c}=\left\{(S, t) \in \overline{\mathbb{W}}_{c, \Theta} \times(-\pi, \pi): \theta(S)=t\right\}$. Denote

$$
\Omega_{0}:=\left\{(S, t) \in \overline{\mathbb{W}}_{c, \Theta}: w\left(S, P_{t}\right) \geq \frac{1}{12}+\frac{3}{4} c\right\},
$$

then $\Omega^{c} \subset \subset \Omega_{0}$ since $\theta(S)=t$ implies $w\left(S, P_{t}\right)=r \geq c>\frac{1}{12}+\frac{3}{4} c$. For all $(S, t) \in \Omega_{0}$, $1-w\left(S, P_{t}\right) \leq 1-\left(\frac{1}{12}+\frac{3}{4} c\right)=\frac{11}{12}-\frac{3}{4} c$ and hence $\varphi\left(1-w\left(S, P_{t}\right)\right)=0$. Noting that $w\left(S, P_{t}\right)>0$ implies $|\theta-t|<\pi$, we have $1-w\left(S, P_{t}\right) \in\left[m_{S}, M_{S}\right)$, and moreover

$$
\Psi_{t}\left(S, 1-w\left(S, P_{t}\right)\right)=r \cos (\theta-t)+\left(1-w\left(S, P_{t}\right)\right)-1=0 .
$$

Therefore

$$
\tilde{H}(S, t)=1-w\left(S, P_{t}\right) \quad \text { for all }(S, t) \in \Omega_{0}
$$

and the smoothness of $\tilde{H}$ follows.

Now we put

$$
U_{t}:=\left\{S \in \overline{\mathbb{W}}_{c, \Theta}: w\left(S, P_{t}\right) \geq \frac{1}{12}+\frac{3}{4} c\right\},
$$

then obviously $0 \leq \tilde{H}(S, t) \leq \frac{11}{12}-\frac{3}{4} c$ whenever $S \in U_{t}$. On the other hand, for arbitrary $S \in \overline{\mathbb{W}}_{c, \Theta} \backslash U_{t}$, one of the following two cases has to occur: (I) $|\theta(S)-t| \geq \pi$; (II) $|\theta(S)-t| \leq \pi$ and $w\left(S, P_{t}\right)<\frac{1}{12}+\frac{3}{4} c$. If Case (I) holds, then $\tilde{H}(S, t) \geq m_{S}>$ $\frac{11}{12}-\frac{3}{4} c$. For the second case, since

$$
\Psi_{t}\left(s, \frac{11}{12}-\frac{3}{4} c\right)=r \cos (\theta-t)+\frac{11}{12}-\frac{3}{4} c-1<0,
$$

one can deduce that $\tilde{H}(S, t)>\frac{11}{12}-\frac{3}{4} c$ due to the monotonicity of $\Psi_{t}$ with respect to the $u$ variable. Therefore

$$
U_{t}=\left\{S \in \overline{\mathbb{W}}_{c, \Theta}: \tilde{H}(S, t) \leq \frac{11}{12}-\frac{3}{4} c\right\} .
$$


Similarly

$$
\operatorname{int}\left(U_{t}\right)=\left\{S \in \overline{\mathbb{W}}_{c, \Theta}: \tilde{H}(S, t)<\frac{11}{12}-\frac{3}{4} c\right\}
$$

It is easily seen from (6.19) and (6.21) that

$\operatorname{Hess} \tilde{H}(\cdot, t) \geq\left(\frac{1}{12}+\frac{3}{4} c\right)\left(-\operatorname{Hess} \log w\left(\cdot, P_{t}\right)+d \log w\left(\cdot, P_{t}\right) \otimes d \log w\left(\cdot, P_{t}\right)\right) \quad$ on $U_{t}$.

For each $a \geq \frac{11}{12}-\frac{3}{4} c$ and $S \in \overline{\mathbb{W}}_{c, \Theta}$ satisfying $\theta(S)>t, \tilde{H}(S, t)=a$ if and only if

$$
0=\Psi_{t}(S, a)=r \cos (\theta-t-\varphi(a))+a-\varphi(a)-1
$$

i.e.

$$
w\left(S, P_{t+\varphi(a)}\right)=1+\varphi(a)-a
$$

with

Hence

$$
\frac{1}{12}+\frac{3}{4} c \geq 1+\varphi(a)-a \geq 1+\lim _{u \rightarrow+\infty}(\varphi(u)-u)=\frac{1}{6}+\frac{1}{2} c>\frac{1}{3}
$$

$$
N_{t, a}^{+}:=\left\{S \in \overline{\mathbb{W}}_{c, \Theta}: \theta(S)>t, \tilde{H}(S, t)=a\right\}
$$

overlaps the level set $\left\{S \in \mathbf{G}_{n, m}:-\log w\left(S, P_{t+\varphi(a)}\right)=-\log (1+\varphi(a)-a)\right\}$.

From (6.16) we have $\tilde{\Psi}_{t}:=\Psi_{t}(S, \tilde{H}(S, t)) \equiv 0$. Differentiating both sides implies

$$
0=\nabla_{\nu} \tilde{\Psi}_{t}=\nabla_{\nu} \Psi_{t}(\cdot, a)+\left(\partial_{2} \Psi_{t}\right) \nabla_{\nu} \tilde{H}(\cdot, t) \quad \text { on } N_{t, a}^{+}
$$

with $\nu$ the unit normal vector field on $N_{t, a}^{+}$. Noting that $\Psi_{t}(\cdot, a)=w\left(\cdot, P_{t+\varphi(a)}\right)-$ $(1+\varphi(a)-a)$ and $\partial_{2} \Psi_{t}>0$, (6.26) tells us $\nabla \tilde{H}(\cdot, t)$ and $-\nabla \log w\left(\cdot, P_{t+\varphi(a)}\right)$ are both nonzero normal vector fields on $N_{t, a}^{+}$, pointing in the same direction. The compactness of $\bigcup_{a \geq \frac{11}{12}-\frac{3}{4} c} N_{t, a}^{+}$and $\Theta$ implies the existence of a positive constant $C_{8}$ not depending on $a$ and $t$, such that

$$
\frac{|\nabla \tilde{H}(\cdot, t)|}{\left|\nabla \log w\left(\cdot, P_{t+\varphi(a)}\right)\right|} \geq C_{8} \quad \text { on } N_{t, a}^{+} .
$$

Hence applying Lemma 4.1 gives

$$
\text { Hess } \tilde{H}(\cdot, t) \geq-C_{8} \log w\left(\cdot, P_{t+\varphi(a)}\right)-\varepsilon g+\lambda d \tilde{H}(\cdot, t) \otimes d \tilde{H}(\cdot, t) \quad \text { on } N_{t, a}^{+}
$$

with $g$ being the canonical metric on $\mathbf{G}_{n, m}, \varepsilon$ being a positive constant to be chosen and $\lambda$ denoting a continuous nonpositive function depending on $\varepsilon$.

Similarly,

$$
N_{t, a}^{-}:=\left\{S \in \overline{\mathbb{W}}_{c, \Theta}: \theta(S)<t, \tilde{H}(S, t)=a\right\}
$$

overlaps the level set $\left\{S \in \mathbf{G}_{n, m}: \log w\left(S, P_{t-\varphi(a)}\right)=-\log (1+\varphi(a)-a)\right\}$ for each $a \geq \frac{11}{12}-\frac{3}{4} c$. On it $\nabla \tilde{H}(\cdot, t)$ and $-\nabla \log w\left(\cdot, P_{t-\varphi(a)}\right)$ are both nonzero normal vector fields pointing in the same direction. Again using Lemma 4.2 yields

$$
\operatorname{Hess} \tilde{H}(\cdot, t) \geq-C_{8} \log w\left(\cdot, P_{t-\varphi(a)}\right)-\varepsilon g+\lambda d \tilde{H}(\cdot, t) \otimes d \tilde{H}(\cdot, t) \quad \text { on } N_{t, a}^{-} \text {. }
$$


Let $M$ be a submanifold in $\mathbb{R}^{n+m}$ with parallel mean curvature whose Gauss image is contained in $\overline{\mathbb{W}}_{c, \Theta}$, denote

$$
\tilde{h}(\cdot, t)=\exp \left(\mu_{0} \tilde{H}(\cdot, t)\right) \circ \gamma
$$

where $\mu_{0}$ is a positive constant to be chosen. Once $\gamma(x) \in U_{t}$, combining (6.23) and (3.23) gives

$$
\begin{aligned}
& \mu_{0}^{-1} \tilde{h}(\cdot, t)^{-1} \tilde{h}(\cdot, t) \\
\geq & -\left(\frac{1}{12}+\frac{3}{4} c\right) \Delta\left(\log w\left(\cdot, P_{t}\right) \circ \gamma\right)+\left(\frac{1}{12}+\frac{3}{4} c+\mu_{0}\right)\left|\nabla\left(\log w\left(\cdot, P_{t}\right) \circ \gamma\right)\right|^{2} \\
\geq & \left(\frac{1}{12}+\frac{3}{4} c\right) C_{1}|B|^{2}+\mu_{0}\left|\nabla\left(\log w\left(\cdot, P_{t}\right) \circ \gamma\right)\right|^{2}
\end{aligned}
$$

at $x \in M$, where $C_{1}$ is a positive constant depending only on $c$. If $\gamma(x) \in N_{t, a}^{+}$, based on (6.28) and (3.23), one can proceed as in the proof of Proposition 4.2 to obtain

$\mu_{0}^{-1} \tilde{h}(\cdot, t)^{-1} \Delta \tilde{h}(\cdot, t) \geq\left(C_{8} C_{1}-\varepsilon\right)|B|^{2}+\left(C_{8}^{2}\left|\lambda+\mu_{0}\right|-C_{8}\right)\left|\nabla\left(\log w\left(\cdot, P_{t+\varphi(a)}\right) \circ \gamma\right)\right|^{2}$

at $x$. Similarly, once $\gamma(x) \in N_{t, a}^{-}$,

$\mu_{0}^{-1} \tilde{h}(\cdot, t)^{-1} \Delta \tilde{h}(\cdot, t) \geq\left(C_{8} C_{1}-\varepsilon\right)|B|^{2}+\left(C_{8}^{2}\left|\lambda+\mu_{0}\right|-C_{8}\right)\left|\nabla\left(\log w\left(\cdot, P_{t-\varphi(a)}\right) \circ \gamma\right)\right|^{2}$.

Now we put

$$
\varepsilon:=C_{8} C_{1}, \quad \mu_{0}:=\sup |\lambda|+C_{8}^{-1}
$$

(where $\sup |\lambda|<+\infty$, since $\lambda$ is a continuous function on a compact set $\left(\overline{\mathbb{W}}_{c, \Theta} \times\right.$ $\left.\Theta) \backslash \operatorname{int}\left(\Omega_{0}\right)\right)$, then from (6.31)-(6.33) we see $\tilde{h}$ is a subharmonic function on $M$. Therefore

$$
H(\cdot, t):=\frac{\exp \left(\mu_{0} \tilde{H}(\cdot, t)\right)-1}{\exp \left(\left(\frac{11}{12}-\frac{3}{4} c\right) \mu_{0}\right)-1}
$$

are required functions satisfying (i)-(iii).

Remark 6.1. The auxiliary function $\varphi$ in the above proof can be easily obtained from the standard bump function. Choose $\xi_{1}$ to be a nonnegative smooth function on $\mathbb{R}$, whose support is $\left(\frac{11}{12}-\frac{3}{4} c, \frac{3}{4}-\frac{1}{4} c\right)$, then

$$
\xi_{2}(u):=\frac{\int_{\frac{11}{12}-\frac{3}{4} c}^{u} \xi_{1}}{\int_{\frac{11}{12}-\frac{3}{4} c}^{\frac{3}{4}-\frac{1}{4} c} \xi_{1}}
$$

is a smooth function on $\mathbb{R}$ satisfying $0 \leq \xi_{2} \leq 1, \xi_{2}(u)=0$ whenever $u \leq \frac{11}{12}-\frac{3}{4}$ c and $\xi_{2}(u)=1$ whenever $u \geq \frac{3}{4}-\frac{1}{4} c . \alpha \in(0,+\infty) \mapsto \int_{\frac{11}{12}-\frac{3}{4} c}^{\frac{3}{4}-\frac{1}{4} c} \xi_{2}^{\alpha}$ is a strictly decreasing function, which converges to 0 as $u \rightarrow+\infty$ and converges to $-\frac{1}{6}+\frac{1}{2}$ c as $u \rightarrow 0$. There exists a unique $\beta \in(0,+\infty)$, such that

$$
\int_{\frac{11}{12}-\frac{3}{4} c}^{\frac{3}{4}-\frac{1}{4} c} \xi_{2}^{\beta}=-\frac{1}{12}+\frac{1}{4} c .
$$


Then

$$
\varphi(u):=\int_{0}^{u} \xi_{2}^{\beta}
$$

is the required auxiliary function.

Based on Lemma 6.1 and Proposition 6.2 6.3, one can derive a Gauss image shrinking property for submanifolds with parallel mean curvature as in [16] [17]:

Lemma 6.2. Let $M^{n}$ be a submanifold in $\mathbb{R}^{n+m}(n \geq 3)$ with parallel mean curvature. Assume there is a distance function $d$ on $M$, the metric ball $B_{4 R_{0}}\left(x_{0}\right)$ given by $d$ satisfies DVP-condition for some $x_{0} \in M$ and $R_{0} \in(0,+\infty]$, and the Gauss image of $B_{4 R_{0}}\left(x_{0}\right)$ is contained in a compact set $K \subset \mathbb{W}_{1 / 3}$. Then there exists a constant $\delta_{1} \in(0,1)$, depending only on $K, K_{1}, K_{2}$, not depending on $x_{0}$ and $R_{0}$, such that the image of $B_{\delta_{1} R_{0}}\left(x_{0}\right)$ under the Gauss map is contained in $\left\{S \in \mathbf{G}_{n, m}: w(S, P) \geq \frac{1}{12}+\frac{3}{4} c\right\}$ for some $P \in \mathbf{G}_{n, m}$, where $c:=\inf _{K} r$.

Lemma 6.3. Let $M^{n}$ be a minimal submanifold in $\mathbb{R}^{n+m}(n \geq 3)$ with rank $(\gamma) \leq 2$ ( $\gamma$ denotes the Gauss map). Assume there is a distance function $d$ on $M$, the metric ball $B_{4 R_{0}}\left(x_{0}\right)$ given by $d$ satisfies the DVP-condition for some $x_{0} \in M$ and $R_{0} \in(0,+\infty]$, and the Gauss image of $B_{4 R_{0}}\left(x_{0}\right)$ is contained in a compact set $K \subset \mathbb{W}_{0}$. Then there exists a constant $\delta_{1} \in(0,1)$, depending only on $K, K_{1}, K_{2}$, not depending on $x_{0}$ and $R_{0}$, such that the image of $B_{\delta_{1} R_{0}}\left(x_{0}\right)$ under the Gauss map is contained in $\left\{S \in \mathbf{G}_{n, m}: w(S, P) \geq \frac{3}{4} c\right\}$ for some $P \in \mathbf{G}_{n, m}$, where $c:=\inf _{K} r$.

Proof. As above, we only prove Lemma 6.2.

Since $K \subset \mathbb{W}_{1 / 3}$ is compact, $c:=\inf _{K} r>\frac{1}{3}$ and $\Theta:=\{t \in(-\pi, \pi): t=$ $\theta(S)$ for some $S \in K\}$ is a compact subset of $(-\pi, \pi)$. Obviously $K \subset \overline{\mathbb{W}}_{c, \Theta}$.

By Lemma 6.1, $\overline{\mathbb{W}}_{c, \Theta}$ is diffeomorphic to $\Theta \times \overline{\mathbb{D}}_{c^{-1}-1}^{n m-1}$. Thus $\overline{\mathbb{W}}_{c, \Theta}$ can be seen as a bounded convex domain in $\mathbb{R}^{n m}$ equipped with the induced metric. The eigenvalues of the metric matrices are bounded from below by $K_{3}$ and from above by $K_{4}$, where $K_{3}, K_{4}$ are positive constants depending only on $K$.

The Ruh-Vilms Theorem [20] implies that $\gamma$ is a harmonic function. Putting $u=\gamma$ in (6.2) gives $|d u|^{2}=|B|^{2}$. Let $\{H(\cdot, t): t \in \Theta\}$ be a family of smooth functions on $\overline{\mathbb{W}}_{c, \Theta}$ as constructed in Proposition 6.2. Choosing one of the functions as a test function in (6.2) yields

$$
H(\gamma(x), t) \leq H\left(\bar{\gamma}_{R}, t\right)+C_{7} C_{9}\left(\frac{R^{2}}{V\left(x_{0}, R\right)} \int_{B_{R}\left(x_{0}\right)}|B|^{2} * 1\right)^{\frac{1}{2}} .
$$

for arbitrary $R \leq R_{0}$ and all $x \in B_{\frac{R}{4}}$. Here

$$
C_{9}:=\sup _{\Theta} \sup _{\overline{\mathbb{W}}_{c, \Theta}}|\nabla H(\cdot, t)|
$$

and the last term in (6.2) has been thrown out, since $H(\cdot, t) \circ \gamma$ is a subharmonic function. 
For arbitrary $S \in \overline{\mathbb{W}}_{c, \Theta}, w\left(S, P_{\theta(S)}\right)=r(S) \geq c>\frac{3}{4} c+\frac{1}{12}$, which implies $H(S, \theta(S))<1$ by Proposition $6.2\left(\right.$ ii). Since $\overline{\mathbb{W}}_{c, \Theta}$ is compact, there exists a positive constant $\varepsilon_{1}$, such that

$$
H(S, \theta(S)) \leq 1-\varepsilon_{1} \quad \text { for all } S \in \overline{\mathbb{W}}_{c, \Theta} .
$$

By Lemma 5.3 (curvature estimates), if we denote

$$
\delta_{1}:=\frac{1}{4} \exp \left(-C_{6} C_{7}^{2} C_{9}^{2} \varepsilon_{1}^{-2}\right)
$$

then there exists $R \in\left[4 \delta_{1} R_{0}, R_{0}\right]$, such that

$$
\frac{R^{2}}{V\left(x_{0}, R\right)} \int_{B_{R}\left(x_{0}\right)}|B|^{2} * 1 \leq C_{7}^{-2} C_{9}^{-2} \varepsilon_{1}^{2}
$$

Letting $t=\theta\left(\bar{\gamma}_{R}\right)$ and substituting (6.38) and (6.40) into (6.36) gives

$$
H\left(\gamma(x), \theta\left(\bar{\gamma}_{R}\right)\right) \leq H\left(\bar{\gamma}_{R}, \theta\left(\bar{\gamma}_{R}\right)\right)+C_{7} C_{9}\left(C_{7}^{-2} C_{9}^{-2} \varepsilon_{1}^{2}\right)^{\frac{1}{2}} \leq 1-\varepsilon_{1}+\varepsilon_{1}=1
$$

for all $x \in B_{\frac{R}{4}}\left(x_{0}\right)$. Hence by Proposition 6.2(ii),

$$
\gamma\left(B_{\delta_{1} R_{0}}\left(x_{0}\right)\right) \subset \gamma\left(B_{\frac{R}{4}}\left(x_{0}\right)\right) \subset\left\{S \in \mathbf{G}_{n, m}: w\left(S, P_{\theta\left(\bar{\gamma}_{R}\right)}\right) \geq \frac{1}{12}+\frac{3}{4} c\right\} .
$$

Given $\gamma\left(B_{\delta_{1} R_{0}}\left(x_{0}\right)\right) \subset\left\{S \in \mathbf{G}_{n, m}: w(S, P) \geq \frac{1}{12}+\frac{3}{4} c>\frac{1}{3}\right\}$, one can start an iteration as in [17] to get a-priori estimates for the Gauss image:

Lemma 6.4. Let $M^{n}$ be a submanifold in $\mathbb{R}^{n+m}(n \geq 3)$ with parallel mean curvature. Assume there is a distance function $d$ on $M$, the metric ball $B_{4 R_{0}}\left(x_{0}\right)$ defined by $d$ satisfies the DVP-condition for some $x_{0} \in M$ and $R_{0} \in(0,+\infty]$, and the Gauss image of $B_{4 R_{0}}\left(x_{0}\right)$ is contained in a compact set $K \subset \mathbb{W}_{1 / 3}$. Then for arbitrary $\varepsilon>0$, there exists a constant $\delta_{2} \in(0,1)$, depending only on $K, K_{1}, K_{2}, \varepsilon$, not depending on $x_{0}$ and $R_{0}$, such that

$$
w\left(\gamma(x), \gamma\left(x_{0}\right)\right) \geq 1-\varepsilon \quad \text { on } B_{\delta_{2} R_{0}}\left(x_{0}\right) .
$$

In particular, if $M$ is a minimal submanifold with $\operatorname{rank}(\gamma) \leq 2$, then the condition on the Gauss image can be relaxed to $\gamma\left(B_{4 R_{0}}\left(x_{0}\right)\right) \subset K \subset \mathbb{W}_{0}$.

Letting $R_{0} \rightarrow+\infty$ we arrive at a Bernstein type theorem.

Theorem 6.1. Let $M^{n}$ be a submanifold in $\mathbb{R}^{n+m}(n \geq 3)$ with parallel mean curvature. Assume there is a distance function $d$ on $M$, such that $M$ satisfies the $D V P$-condition and the diameter of $M$ with respect to $d$ is infinite; and there exist $P, Q \in \mathbf{G}_{n, m}$ that are $S$-orthogonal to each other, such that $(w(\gamma(x), P), w(\gamma(x), Q))$ is contained in a compact subset $K$ of $\overline{\mathbb{D}} \backslash\left(\overline{\mathbb{D}}_{1 / 3} \cup\{(a, 0): a \leq 0\}\right)$ for all $x \in M$. Then $M$ has to be an affine linear subspace.

In particular, if $M$ is a minimal submanifold with $\operatorname{rank}(\gamma) \leq 2$, then the assumptions on the Gauss image can be replaced by $(w(\gamma(x), P), w(\gamma(x), Q)) \in K \subset \overline{\mathbb{D}} \backslash\{(a, 0)$ : $a \leq 0\}$. 
The above general Theorem can be applied to graphic submanifolds as follows.

Theorem 6.2. Let $z^{\alpha}=f^{\alpha}\left(x^{1}, \cdots, x^{n}\right), \alpha=1, \cdots, m$, be smooth functions defined everywhere in $\mathbb{R}^{n}(n \geq 3, m \geq 2)$, such that their graph $M=(x, f(x))$ is a submanifold with parallel mean curvature in $\mathbb{R}^{n+m}$. Suppose that there exist $\beta_{0}<+\infty$ and $\beta_{1}<3$, such that

$$
\Delta_{f}:=\left[\operatorname{det}\left(\delta_{i j}+\sum_{\alpha} \frac{\partial f^{\alpha}}{\partial x^{i}} \frac{\partial f^{\alpha}}{\partial x^{j}}\right)\right]^{\frac{1}{2}} \leq \beta_{0}
$$

and

$$
\Delta_{f} \leq \beta_{1}\left(1+\left(\frac{\partial f^{1}}{\partial x^{1}}\right)^{2}\right)^{\frac{1}{2}}
$$

Then $f^{1}, \cdots, f^{m}$ have to be affine linear (representing an affine $n$-plane).

Proof. $F: \mathbb{R}^{n} \rightarrow M$ defined by

$$
x \mapsto(x, f(x))
$$

is obviously a diffeomorphism. Thus $M$ can be viewed as an $n$-dimensional Euclidean space equipped with metric $g=g_{i j} d x^{i} d x^{j}$. Here

$$
g_{i j}=\left\langle F_{*} \frac{\partial}{\partial x^{i}}, F_{*} \frac{\partial}{\partial x^{j}}\right\rangle=\left\langle\varepsilon_{i}+\frac{\partial f^{\alpha}}{\partial x^{i}} \varepsilon_{n+\alpha}, \varepsilon_{j}+\frac{\partial f^{\alpha}}{\partial x^{j}} \varepsilon_{n+\alpha}\right\rangle=\delta_{i j}+\frac{\partial f^{\alpha}}{\partial x^{i}} \frac{\partial f^{\alpha}}{\partial x^{j}}
$$

with $\left\{\varepsilon_{i}, \varepsilon_{n+\alpha}\right\}$ being the canonical orthonormal basis of $\mathbb{R}^{n+m}$. Let $D f:=\left(\frac{\partial f^{\alpha}}{\partial x^{i}}\right)$ be an $(n \times m)$-matrix valued function on $\mathbb{R}^{n}$, then

$$
\left(g_{i j}\right)=I_{n}+D f(D f)^{T} .
$$

Hence $\left(g_{i j}\right) \geq I_{n}$. In conjunction with $\operatorname{det}\left(g_{i j}\right)=\Delta_{f}^{2} \leq \beta_{0}^{2}$, we can deduce that all the eigenvalues of $\left(g_{i j}\right)$ take values between 1 and $\beta_{0}^{2}$. Denote $d: M \times M \rightarrow \mathbb{R}$

$$
d(F(x), F(y))=|x-y|,
$$

then the diameter of $M$ is $+\infty$ and it has been shown in Remark 5.1] that $M$ satisfies a DVP-condition.

Let $P, Q \in \mathbf{G}_{n, m}$ whose Plücker coordinates are $\varepsilon_{1} \wedge \cdots \wedge \varepsilon_{n}$ and $\varepsilon_{n+1} \wedge \varepsilon_{2} \wedge \cdots \wedge \varepsilon_{n}$, respectively. Obviously, $P, Q$ are S-orthogonal to each other. Denote by $\psi$ the Plücker embedding, then

$$
\psi \circ \gamma=\left(\varepsilon_{1}+\frac{\partial f^{\alpha}}{\partial x^{1}} \varepsilon_{n+\alpha}\right) \wedge \cdots \wedge\left(\varepsilon_{n}+\frac{\partial f^{\alpha}}{\partial x^{n}} \varepsilon_{n+\alpha}\right)
$$

and by a direct computation,

$$
\begin{aligned}
& w(\gamma, P)=\frac{\langle\psi \circ \gamma, P\rangle}{\langle\psi \circ \gamma, \psi \circ \gamma\rangle^{\frac{1}{2}}\langle P, P\rangle^{\frac{1}{2}}}=\Delta_{f}^{-1} \\
& w(\gamma, Q)=\frac{\langle\psi \circ \gamma, Q\rangle}{\langle\psi \circ \gamma, \psi \circ \gamma\rangle^{\frac{1}{2}}\langle Q, Q\rangle^{\frac{1}{2}}}=\frac{\partial f^{1}}{\partial x^{1}} \Delta_{f}^{-1} .
\end{aligned}
$$

Hence $(w(\gamma, P), w(\gamma, Q)) \in K \subset \overline{\mathbb{D}} \backslash\left(\overline{\mathbb{D}}_{1 / 3} \cup\{(a, 0): a \leq 0\}\right)$ if and only if

$$
\beta_{1}^{-2} \leq w(\gamma, P)^{2}+w(\gamma, Q)^{2}=\left(1+\left(\frac{\partial f^{1}}{\partial x^{1}}\right)^{2}\right) \Delta_{f}^{-2}
$$


for a constant $\beta_{1}<3$. This is equivalent to (6.44). So the Bernstein type result follows from Theorem 6.1.

Remark 6.2. Obviously $\beta_{1}\left(1+\left(\frac{\partial f^{1}}{\partial x^{1}}\right)^{2}\right)^{\frac{1}{2}} \geq \beta_{1}$ on the right hand side of 6.44 . Hence Corollary 6.2 improves Theorem 6.2 in [17]. We note that $\frac{\partial f^{1}}{\partial x^{1}}$ in (6.44) can be replaced by $\frac{\partial f^{\alpha}}{\partial x^{i}}$ for arbitrary $1 \leq \alpha \leq m$ and $1 \leq i \leq n$.

\section{Appendix}

Let $\mathbb{H}$ denote the quaternions with the standard basis $1, i, j, k$ and $\mathbb{C}=\mathbb{R}+$ $\mathbb{R} i, \mathbb{H}=\mathbb{C}+\mathbb{C} j$. Let $q=z_{1}-\bar{z}_{2} j \in \mathbb{H}$ with $z_{1}, z_{2} \in \mathbb{C}$. Then $\bar{q}=\bar{z}_{1}+\bar{z}_{2} j$ and

$$
\begin{aligned}
q i \bar{q} & =\left(z_{1}-\bar{z}_{2} j\right) i\left(\bar{z}_{1}+\bar{z}_{2} j\right) \\
& =\left(\bar{z}_{2} k+z_{1} i\right)\left(\bar{z}_{1}+\bar{z}_{2} j\right) \\
& =\bar{z}_{2} k \bar{z}_{1}+z_{1} i \bar{z}_{1}+\bar{z}_{2} k \bar{z}_{2} j+z_{1} i \bar{z}_{2} j \\
& =z_{1} \bar{z}_{2} k+\left|z_{1}\right|^{2} i-\left|z_{2}\right|^{2} i+z_{1} \bar{z}_{2} k \\
& =\left(\left|z_{1}\right|^{2}-\left|z_{2}\right|^{2}\right) i+2 z_{1} \bar{z}_{2} k
\end{aligned}
$$

which coincides with the usual Hopf map

$$
\begin{aligned}
& \eta: \mathbb{H} \rightarrow \operatorname{Im} \mathbb{H} \\
& \eta=\left(\left(\left|z_{1}\right|^{2}-\left|z_{2}\right|^{2}, 2 z_{1} \bar{z}_{2}\right): \mathbb{R}^{4} \rightarrow \mathbb{R}^{3}\right.
\end{aligned}
$$

with $\eta\left(S^{3}\right) \in S^{2}$. Let

$$
\zeta(x)=s(r) \eta\left(\frac{x}{r}\right)=s(r) r^{-2} \eta(x):=\tilde{s}(r) \eta(x)
$$

with $r=|x|$. It was shown in ([13] Theorem 3.2, p. 135) that

$$
x \rightarrow(x, \zeta(x))
$$

define a coassociative 4 - submanifold in $\mathbb{R}^{7}$ invariant under $S^{3}$, provided

$$
s\left(4 s^{2}-5 r^{2}\right)^{2}=C, \quad C \in \mathbb{R} .
$$

Those are area-minimazing smooth minimal submanifolds except in the case $C=0$. When $C=0$ then $s(r)=\frac{\sqrt{5}}{2} r$ and then $\tilde{s}(r)=\frac{\sqrt{5}}{2 r}$, the function $\zeta(x): \mathbb{R}^{4} \rightarrow \mathbb{R}^{3}$ given by

$$
\zeta=\frac{\sqrt{5}}{2 r} \eta(x)
$$

defines a cone over the entire $\mathbb{R}^{4}$. This was discovered by Lawson and Osserman [18]. This LO-cone shows that Moser's theorem that entire minimal graphs of bounded slope are affine linear cannot be extended to the case of dimension 4 and codimension 3. In this appendix, we compute some important geometric quantities of this remarkable example. 
Put

$$
x=\left(z_{1}, z_{2}\right) \in \mathbb{C}^{2}=\mathbb{R}^{4}, \quad z_{1}=r_{1} e^{i \theta_{1}}, \quad z_{2}=r_{2} e^{i \theta_{2}}, \quad|x|^{2}=r^{2}=r_{1}^{2}+r_{2}^{2} .
$$

The flat metric on $\mathbb{R}^{4}$ reads

$$
d s^{2}=d r_{1}^{2}+r_{1}^{2} d \theta_{1}^{2}+d r_{2}^{2}+r_{2}^{2} d \theta_{2}^{2} .
$$

The orthonormal basis on $T_{x} \mathbb{R}^{4}$ is given by $\left\{e_{0}, e_{1}, e_{2}, e_{3}\right\}$, where

$$
\begin{gathered}
e_{0}=\frac{\partial}{\partial r}=\frac{r_{1}}{r} \frac{\partial}{\partial r_{1}}+\frac{r_{2}}{r} \frac{\partial}{\partial r_{2}}, \quad e_{1}=\frac{r_{2}}{r} \frac{\partial}{\partial r_{1}}-\frac{r_{1}}{r} \frac{\partial}{\partial r_{2}} \\
e_{2}=\frac{r_{2}}{r_{1} r} \frac{\partial}{\partial \theta_{1}}-\frac{r_{1}}{r_{2} r} \frac{\partial}{\partial \theta_{2}}, \quad e_{3}=\frac{1}{r}\left(\frac{\partial}{\partial \theta_{1}}+\frac{\partial}{\partial \theta_{2}}\right) .
\end{gathered}
$$

Now, we have

$$
\eta(x)=\left(r_{1}^{2}-r_{2}^{2}, 2 r_{1} r_{2} e^{i\left(\theta_{1}-\theta_{2}\right)}\right): \mathbb{R}^{4} \rightarrow \mathbb{R}^{3}
$$

Since

$$
\frac{\partial}{\partial r_{1}} \tilde{s}(r)=\tilde{s}^{\prime} \frac{r_{1}}{r}, \quad \frac{\partial}{\partial r_{2}} \tilde{s}(r)=\tilde{s}^{\prime} \frac{r_{2}}{r}
$$

then

$$
\begin{aligned}
& \zeta_{*} e_{0}=\left(\frac{r_{1}}{r} \frac{\partial}{\partial r_{1}}+\frac{r_{2}}{r} \frac{\partial}{\partial r_{2}}\right)(\tilde{s} \eta)=\left(\tilde{s}^{\prime}+\frac{2 \tilde{s}}{r}\right) \eta \\
& \zeta_{*} e_{1}=\frac{r_{2}}{r} \frac{\partial}{\partial r_{1}}(\tilde{s} \eta)-\frac{r_{1}}{r} \frac{\partial}{\partial r_{2}}(\tilde{s} \eta)=\frac{\tilde{s}}{r}\left(4 r_{1} r_{2}, 2\left(r_{2}^{2}-r_{1}^{2}\right) e^{i\left(\theta_{1}-\theta_{2}\right)}\right) .
\end{aligned}
$$

Since

$$
\begin{aligned}
& \zeta_{*} \frac{\partial}{\partial \theta_{1}}=\frac{\partial}{\partial \theta_{1}}(\tilde{s} \eta)=\tilde{s}\left(0,2 i r_{1} r_{2} e^{i\left(\theta_{1}-\theta_{2}\right)}\right), \\
& \zeta_{*} \frac{\partial}{\partial \theta_{2}}=\frac{\partial}{\partial \theta_{2}}(\tilde{s} \eta)=\tilde{s}\left(0,-2 i r_{1} r_{2} e^{i\left(\theta_{1}-\theta_{2}\right)}\right)
\end{aligned}
$$

then

$$
\begin{aligned}
\zeta_{*} e_{2} & =\frac{r_{2}}{r_{1} r} \zeta_{*} \frac{\partial}{\partial \theta_{1}}-\frac{r_{1}}{r_{2} r} \zeta_{*} \frac{\partial}{\partial \theta_{2}}=\tilde{s} r\left(0,2 i e^{i\left(\theta_{1}-\theta_{2}\right)}\right) \\
\zeta_{*} e_{3} & =0
\end{aligned}
$$

Put

$$
\begin{aligned}
& \rho_{0}^{2}=\frac{1}{1+\left|\zeta_{*} e_{0}\right|^{2}}=\frac{1}{1+\left(r^{2} \tilde{s}^{\prime}+2 r \tilde{s}\right)^{2}}, \quad \rho_{1}^{2}=\frac{1}{1+\left|\zeta_{*} e_{1}\right|^{2}}=\frac{1}{1+4 r^{2} \tilde{s}^{2}} \\
& \rho_{2}^{2}=\frac{1}{1+\left|\zeta_{*} e_{2}\right|^{2}}=\frac{1}{1+4 r^{2} \tilde{s}^{2}}, \quad \rho_{3}^{2}=\frac{1}{\left.1+\left|\zeta_{*} e_{3}\right|^{2}\right)}=1
\end{aligned}
$$

Then the Gauss map $\gamma$ for the coassociate $4-$ submanifold is expressed by

$$
\left(e_{0}, \zeta_{*} e_{0}\right) \wedge\left(e_{1}, \zeta_{*} e_{1}\right) \wedge\left(e_{2}, \zeta_{*} e_{2}\right) \wedge\left(e_{3}, \zeta_{*} e_{3}\right)
$$

and the correspoinding $W$-matrix relative to $e_{0} \wedge e_{1} \wedge e_{2} \wedge e_{3}$ is 
The nonzero Jordan angles are

$$
W=\left(\begin{array}{cccc}
\rho_{0} & 0 & 0 & 0 \\
0 & \rho_{1} & 0 & 0 \\
0 & 0 & \rho_{2} & 0 \\
0 & 0 & 0 & 1
\end{array}\right)
$$

$$
\theta_{0}=\arccos \rho_{0}, \theta_{1}=\arccos \rho_{1}, \theta_{2}=\arccos \rho_{2} .
$$

In particular, the Jordan angles of the image under the Gauss map for the $L O$ cone can be obtained by substituting $\tilde{s}=\frac{\sqrt{5}}{2} r^{-1}$ in the above expressions. Those are the following constants

$$
\theta_{0}=\arccos \frac{2}{3}, \quad \theta_{1}=\theta_{2}=\arccos \frac{\sqrt{6}}{6} .
$$

The $w$-function is identically $\frac{1}{9}$ and hence $v$-function, denoted also by $\Delta_{f}$ for the graphic case, equals 9 . This fact was originally verified by a computer program by Lawson-Osserman in [18]. The LO-cone defined by $\left(f^{1}, f^{2}, f^{3}\right)$ on $\mathbb{R}^{4}$, where

$$
\begin{aligned}
& f^{1}=\frac{\sqrt{5}}{2} \frac{\left(x^{1}\right)^{2}+\left(x^{2}\right)^{2}-\left(x^{3}\right)^{2}-\left(x^{4}\right)^{2}}{\sqrt{\left(x^{1}\right)^{2}+\left(x^{2}\right)^{2}+\left(x^{3}\right)^{2}+\left(x^{4}\right)^{2}}}, \\
& f^{2}=\frac{\sqrt{5}}{2} \frac{2\left(x^{1} x^{3}+x^{2} x^{4}\right)}{\sqrt{\left(x^{1}\right)^{2}+\left(x^{2}\right)^{2}+\left(x^{3}\right)^{2}+\left(x^{4}\right)^{2}}}, \\
& f^{3}=\frac{\sqrt{5}}{2} \frac{2\left(x^{1} x^{3}-x^{2} x^{4}\right)}{\sqrt{\left(x^{1}\right)^{2}+\left(x^{2}\right)^{2}+\left(x^{3}\right)^{2}+\left(x^{4}\right)^{2}}} .
\end{aligned}
$$

At $x^{3} \neq 0$ and $x^{1}=x^{2}=x^{3}=0$, we have $\frac{\partial f^{2}}{\partial x^{1}}=\sqrt{5}$ and $\left(1+\left(\frac{\partial f^{2}}{\partial x^{1}}\right)^{2}\right)^{\frac{1}{2}}=\sqrt{6}$.

\section{REFERENCES}

1. W.Allard: On the first variation of a varifold. Ann.Math. 95 (1972), 417-491

2. J.L.M.Barbosa: An extrinsic rigidity theorem for minimal immersion from $S^{2}$ into $S^{n}$. J. Diff. Geom. 14(3) (1980), 355-368.

3. E. Bombieri, E. Giusti: Harnack's inequality for elliptic differential equations on minimal surfaces. Invent. Math. 15(1972), 24-46.

4. P. Buser: A note on the isoperimetric constant. Ann. Scient. Ec. Norm. Sup. 15(1982), 213-230.

5. S. S. Chern and R. Osserman: Complete minimal surfaces in Euclidean $n$-space. J. d'Anal. Math. 19(1967), 15-34.

6. D. Fischer-Colbrie: Some rigidity theorems for minimal submanifolds of the sphere, Acta math. 145(1980), 29-46.

7. M. Giaquinta, E. Giusti: On the regularity of the minima of variational integrals, Acta Math. 148 (1982), 31-46.

8. M. Giaquinta, S. Hildebrandt: A priori estimates for harmonic mappings, J. reine angew. Math. 336 (1982), 124-164.

9. M. Grüter, K. Widman: The Green function for uniformly elliptic equations, Manuscripta Math. 37(1982), 303-342.

10. R. Gulliver, J. Jost: Harmonic maps which solve a free-boundary problem, J. reine angew. Math. 381 (1987), 61-89. 
11. S. Hildebrandt, H. Kaul, K.O. Widman: An existence theorem for harmonic mappings of Riemannian manifolds, Acta Math. 138 (1977), 1-16.

12. S. Hildebrandt, J. Jost, K. Widman: Harmonic mappings and minimal submanifolds, Invent. math. 62(1980), 269-298.

13. R. H. Harvey and B. Lawson: Calibrated geometry, Acta Math. 148 (1982), 47-157.

14. J. Jost: Generalized Dirichlet forms and harmonic maps, Calc. Var. PDE 5(1997), 1-19.

15. J. Jost, Y. L. Xin: Bernstein type theorems for higher codimension, Calc.Var. and Partial Differential Equations 9 (1999), 277-296.

16. J. Jost, Y. L. Xin and Ling Yang: The regularity of harmonic maps into spheres and applications to Bernstein problems. arXiv:0912.0447v1. to appear in JDG.

17. J. Jost, Y. L. Xin and Ling Yang: The Gauss image of entire graphs of higher codimension and Bernstein type theorems. arXiv: 1009.3901v1. to appear in Calc. Var. and and Partial Differential Equations.

18. H. B. Lawson and R. Osserman: Non-existence, non-uniqueness and irregularity of solutions to the minimal surface system. Acta math. 139(1977), 1-17.

19. J. Moser: On Harnack's theorem for elliptic differential equations, Comm. Pure Appl. Math. 14(1961), 577-591.

20. E. A. Ruh and J. Vilms: The tension field of Gauss maps, Trans. AMS. 149(1970), 569-573.

21. J. Simons: Minimal varieties in Riemannian manifolds. Ann. Math. 88 (1968), 62-105.

22. Yung-Chow Wong: Differential geometry of Grassmann manifolds, Proc. N.A.S. 57 (1967), 589-594.

23. Yuanlong Xin: Minimal submanifolds and related topics, World Scientic Publ. 2003.

24. Y. L. Xin: Bernstein type theorems without graphic condition, Asian J. Math. 9(1), (2005), 31-44.

25. Y. L. Xin: Curvature estimates for submanifolds with prescribed Gauss image and mean curvature, Calc. Var. and Partial Differential Equations 37(3-4) (2010), 385-405.

26. Y. L. Xin and Ling Yang: Convex functions on Grassmannian manifolds and Lawson-Osserman problem, Adv. Math. 219(4), (2008), 1298-1326.

Max Planck Institute for Mathematics in the Sciences, Inselstr. 22, 04103 LEIPZIG, GERMANY

Department of Mathematics and Computer Science, University of Leipzig, 04109 Leipzig, Germany.

E-mail address: jost@mis.mpg.de

Institute of Mathematics, Fudan University, Shanghai 200433, China.

E-mail address: ylxin@fudan.edu.cn

Institute of Mathematics, Fudan University, Shanghai 200433, China.

E-mail address: lingyang@fudan.edu.cn 